ARTICLE

https://doi.org/10.1038/s41467-019-10823-8

\title{
Monoallelic expression and epigenetic inheritance sustained by a Trypanosoma brucei variant surface glycoprotein exclusion complex
}

\author{
Joana Faria', Lucy Glover ${ }^{1,2}$, Sebastian Hutchinson (D) ${ }^{1,3}$, Cordula Boehm¹, Mark C. Field (D) ${ }^{1}$ \& David Horn (D) ${ }^{1}$
}

The largest gene families in eukaryotes are subject to allelic exclusion, but mechanisms underpinning single allele selection and inheritance remain unclear. Here, we describe a protein complex sustaining variant surface glycoprotein (VSG) allelic exclusion and antigenic variation in Trypanosoma brucei parasites. The VSG-exclusion-1 (VEX1) protein binds both telomeric VSG-associated chromatin and VEX2, an ortholog of nonsense-mediated-decay helicase, UPF1. VEX1 and VEX2 assemble in an RNA polymerase-I transcription-dependent manner and sustain the active, subtelomeric VSG-associated transcription compartment. VSG transcripts and VSG coats become highly heterogeneous when VEX proteins are depleted. Further, the DNA replication-associated chromatin assembly factor, CAF-1, binds to and specifically maintains VEX1 compartmentalisation following DNA replication. Thus, the VEX-complex controls VSG-exclusion, while CAF-1 sustains VEX-complex inheritance in association with the active-VSG. Notably, the VEX2-orthologue and CAF-1 in mammals are also implicated in exclusion and inheritance functions. In trypanosomes, these factors sustain a highly effective and paradigmatic immune evasion strategy.

\footnotetext{
${ }^{1}$ The Wellcome Trust Centre for Anti-Infectives Research, School of Life Sciences, University of Dundee, Dow Street, Dundee DD1 5EH, UK. ${ }^{2}$ Present address: Trypanosome Molecular Biology, Department of Parasites and Insect Vectors, Institut Pasteur, 25-28 Rue du Docteur Roux, 75015 Paris, France. ${ }^{3}$ Present address: Trypanosome Cell Biology Unit, INSERM U1201, Department of Parasites and Insect Vectors, Institut Pasteur, 25-28 Rue du Docteur Roux, 75015 Paris, France. Correspondence and requests for materials should be addressed to D.H. (email: d.horn@dundee.ac.uk)
} 
A ntigenic variation and host immune evasion by parasites causing malaria ${ }^{1}$, giardiasis ${ }^{2}$ and African trypanosomiasis ${ }^{3}$ depend upon monoallelic and switchable variant surface antigen expression. Indeed, allelic exclusion governs the expression of many of the largest known gene-families, typically encoding cell-surface proteins in protozoa and mammals, and has major impacts on health and disease. In mammals, odour detection depends upon olfactory receptor exclusion ${ }^{4}$ and B- and T-cell specificity depends upon immunoglobulin and surface receptor exclusion, respectively ${ }^{5}$. Selection of a single allele from a large gene family is thought to be inherently stochastic rather than deterministic and generates tremendous cellular diversity, upon which adaptive mechanisms operate. Notably, the diversitygenerating exclusion systems of protozoan antigenic variation, and the host immune response, are mutually adaptive and in direct competition. Despite intense study, the molecular mechanisms facilitating the selection and maintenance of a single active allele, coordinated with heritable exclusion of all others, remain poorly understood.

The African trypanosome, Trypanosoma brucei, is transmitted among mammalian hosts by tsetse-flies and, due to effective immune evasion, causes chronic and lethal infections, specifically sleeping-sickness in humans and nagana in cattle. In these parasites RNA polymerase-I (pol-I) transcribes a single, dominant, telomeric variant surface glycoprotein (VSG) gene ${ }^{3}$ as part of a polycistronic transcription unit. Pol-I transcription initiates at several sites but this is attenuated at all but one site ${ }^{6}$. T. brucei can switch to activate a new telomeric VSG expression site (ESs) but, significantly, switching occurs at low-frequency and all but one of the ESs typically remain 'silent', despite possessing all ciselements required for expression?

The active VSG-ES is associated with an extranucleolar pol-I transcription factory, known as the expression site body (ESB) $)^{8,9}$. This VSG-ES is depleted of nucleosomes ${ }^{10,11}$ and nucleasehypersensitive chromatin persists at this locus even if transcription is blocked ${ }^{12}$. An HMG chromatin protein is enriched at both the ESB and the nucleolus ${ }^{12,13}$, and a focus of SUMOylation associates with the $\mathrm{ESB}^{14}$. In addition, histones ${ }^{15}$ and histone variants ${ }^{16}$, telomere-binding proteins, a histone H3-K76 methyltransferase, a histone deacetylase, a chromatin remodeler, histone chaperones, nuclear lamina components, cohesin and the inositol phosphate pathway all impact VSG-ES silencing to varying degrees (reviewed in ref. ${ }^{17}$ ). These factors may impact chromatindependent silencing or transcriptional permissivity but, notably, a direct role in gene selection has not been demonstrated. More recently, the first factor specifically enriched in association with the active VSG-ES, VSG exclusion 1 (VEX1), was identified ${ }^{17}$.

Here, we identify and characterise a VEX1-VEX2 complex. The complex assembles a sub-nuclear domain in a transcriptiondependent manner and maintains VSG allelic exclusion by negatively controlling transcription of other telomeric VSGs. Inheritance of VSG exclusion requires maintenance of the VEXcomplex during S-phase, which depends upon the conserved chromatin assembly factor, CAF-1.

\section{Results}

A subtelomere- and VSG-associated VEX-complex. We previously described T. brucei VEX1 (Tb927.11.16920), the only known protein specifically enriched in association with the $\mathrm{ESB}^{17}$. VEX1, therefore, is at the heart of the nuclear subdomain meditating antigenic variation in trypanosomes, but VEX1 lacks orthologs in other cell types, it remained unclear why VSG-exclusion was only partially perturbed following VEX1-knockdown and it remained unclear whether other factors were involved in VSG-exclusion.
To identify VEX1 chromatin interactions, we affinity-purified

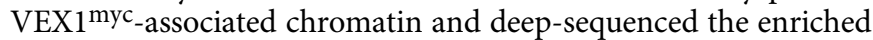
DNA. Reads were accurately aligned to individual VSG-ESs by uniqueness filtering, using $\mathrm{MapQ}>1^{18}$. An examination of protein coding sequences within the hemizygous subtelomeric $V S G$-ESs ${ }^{7,19}$ revealed VSG-2, the active VSG, as the most enriched gene (Fig. 1a, Supplementary Data 1, sheet 1). We also observed particularly strong enrichment of the region downstream of this gene (Fig. 1a). This is consistent with a focus of VEX1 that is adjacent to, rather than coincident with, the extranucleolar focus of pol-I at the active VSG-ES ${ }^{17}$. Notably, DNA immediately downstream of several silent ES-associated VSGs was also enriched (Fig. 1a, Supplementary Fig. 1). This is also consistent with our model proposing that conserved VSG-associated sequences, including the telomeric repeats, participate in VEX1and homology-dependent VSG-silencing ${ }^{17}$. Thus, chromatin interactions connect VEX1 to the conserved sequences associated with telomeric VSGs.

Next, we affinity-purified VEX1 $\mathrm{GFP}_{\text {-associated proteins using }}$ cryomilling and high-affinity nanobodies. The procedure was carried out initially in insect-stage T. brucei (Supplementary Fig. 2a, b), for which protocols were established ${ }^{20}$, and then in bloodstream form T. brucei (Fig. 1b). Quantitative proteomic analysis (Fig. 1b, Supplementary Fig. 2a, b, Supplementary Data 1, sheet 2) revealed tag-dependent enrichment of green flourescent protein (GFP) and the same set of five proteins in four independent experiments; VEX1 was enriched, as expected, but also Tb927.11.13380, an ortholog of the nonsense-mediated mRNA-decay ATP-dependent superfamily 1-type helicases, UPF1/SMG2/NAM7/Rent121 ${ }^{21}$ (Fig. 1c, Supplementary Fig. 2c-e), now designated VEX2 (predicted $224 \mathrm{kDa}, 2026$ residues). Trypanosomes encode two UPF1-related proteins (Supplementary Fig. 2c). The putative T. brucei canonical UPF1 has been studied but it remains unclear whether classical nonsense-mediated decay operates $^{22}$. VEX2 contains a UPF1-related helicase domain towards the $C$-terminus, including the canonical motifs involved in ATP binding and hydrolysis and nucleic acid binding (Supplementary Fig. 2d). Structural prediction suggested the presence of an $a$-solenoid architecture at the $N$-terminus (Supplementary Fig. 2e), in place of the typical UPF2-interacting domain ${ }^{23}$; a-solenoids are frequently involved in protein-protein or protein-RNA interactions. The other three proteins that displayed tag-dependent enrichment were all three components of chromatin assembly factor 1 (CAF-1; CAF-1a, Tb927.8.3980; CAF-1b, Tb927.10.7050, CAF-1c, Tb927.11.4970; Fig. 1b, c, Supplementary Fig. 2a), the evolutionary conserved heterotrimeric replication-associated histone chaperone ${ }^{24}$. Notably, no pol-I components were enriched.

Super-resolution fluorescence microscopy revealed that, like VEX1, VEX2 localised to a single major sub-nuclear focus (detected in $\sim 80 \%$ of nuclei) that was replicated and segregated at the appropriate phases of the cell cycle (Fig. 1d). Consistent with the proteomics evidence, VEX2-foci co-localised with VEX1 foci (Fig. 1e, Supplementary Movie 1) and the extranucleolar, histonedepleted pol-I compartment (Fig. 1f). We conclude that the VEX1-VEX2-complex is associated with the single VSG transcription compartment.

VEX-complex compartmentalisation is transcription-dependent. VEX1 foci are disrupted in cells treated with transcription inhibitors, indicating that active transcription is required for assembly ${ }^{17,25}$. However, it was unclear from these studies whether VEX1 was degraded or redistributed throughout the nucleoplasm. Protein blotting indicated that neither VEX1 (Fig. 2a) nor VEX2 (Fig. 2b) were degraded following pol-I specific transcription 
a
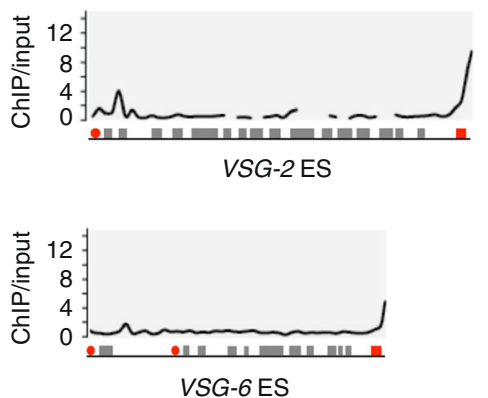

VSG-6 ES
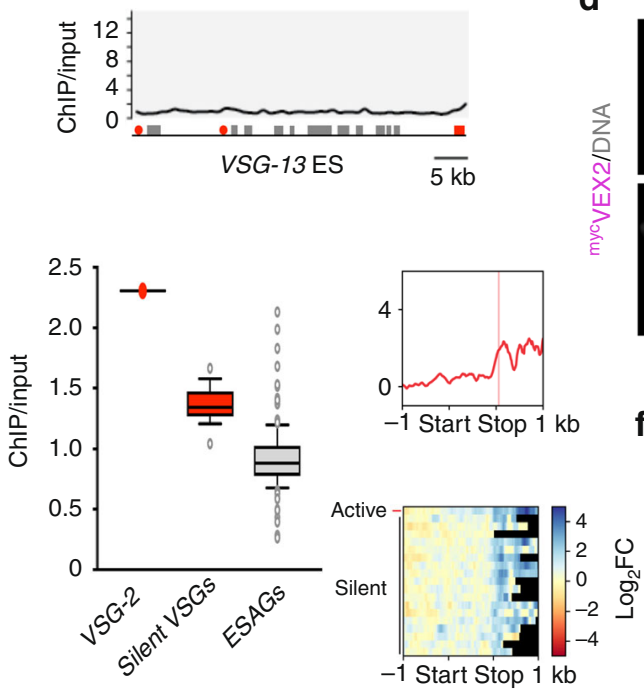

-1 Start Stop $1 \mathrm{~kb}$ b

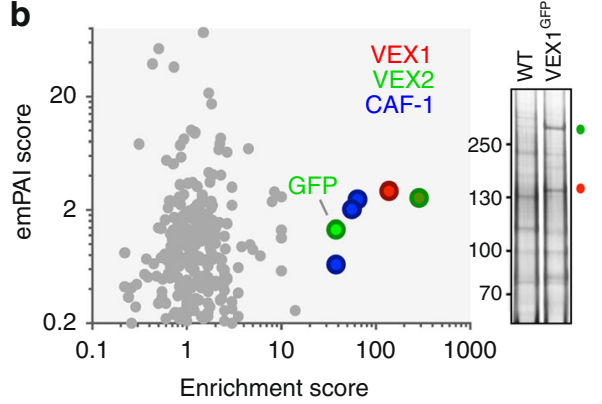

C

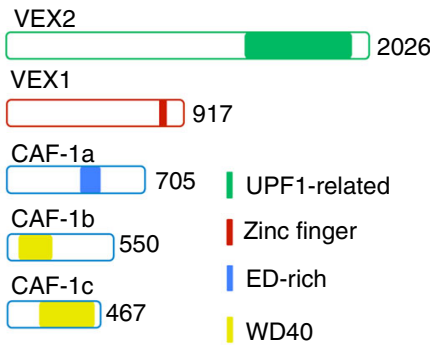

d

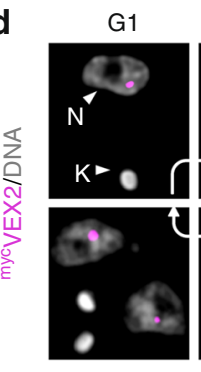

Post-M

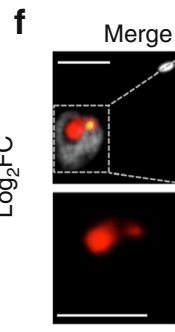

pol-I

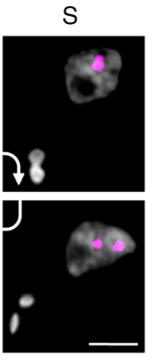

G2

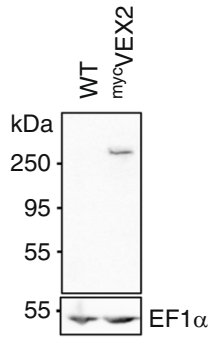

e
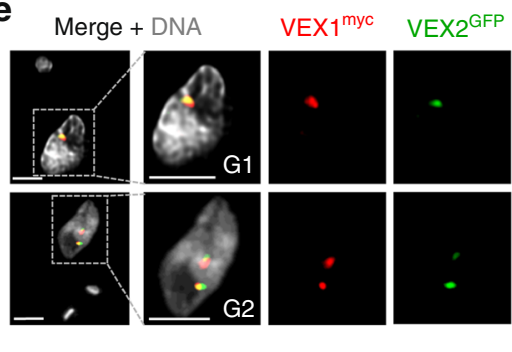

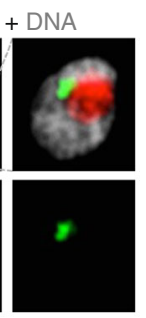

NOG1 mycVEX2

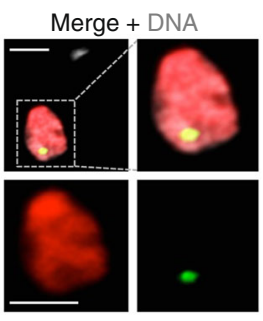

Histone H3 ${ }^{m y c} V E X 2$

Fig. 1 A subtelomere- and VSG-associated VEX-complex. a Affinity purification of VEX1myc-associated chromatin followed by sequencing (ChIP-seq). Enrichment traces over active (VSG-2) and silent (VSG-6, VSG-13) VSG-ESs with 1 kbp non-overlapping bins; red circles, promoters; red boxes, VSGs; grey boxes, expression-site associated genes (ESAGs). Box-plot depicting enrichment of the indicated CDSs. Centre lines show the medians; box limits indicate the 25th and 75th percentiles; whiskers extend from the 10th to the 90th percentile, outliers are shown. Silent VSGs, $n=18 ; E S A G s, n=129$. Metagene plot and the associated heat-map for active and silent telomeric VSGs. The red bar on the metagene plot indicates the location of a highly conserved sequence in the VSG 3'-untranslated region. b VEX1GFP immunoprecipitation and proteomics. GFP-tag dependent enrichment from bloodstream form T. brucei. emPAl, exponentially modified protein abundance index. The inset shows immunoprecipitates and tag-dependent VEX1 (red) and VEX2 (green) bands highlighted in a silver-stained gel. c Schematic representation of VEX1 (Tb927.11.16920), VEX2 (Tb927.11.13380) and CAF-1 subunits (a, Tb927.8.3980; b, Tb927.10.7050; c, Tb927.11.4970) indicating conserved domains and motifs. d Immunofluorescence analysis during the cell cycle and protein-blot analysis of mycVEX2 expression. N nucleus; K kinetoplast (mitochondrial genome). e, $\mathbf{f}$ Immunofluorescence-based colocalisation studies of VEX2 GFP and VEX1myc (e) or a nucleolar (No) and ESB marker (pol-I, largest subunit), a nucleolar marker (NOG1) or Histone H3 (f). In e, G1 or G2 cells are shown. d-f DNA was counter-stained with DAPI; the images correspond to maximal 3D projections of $0.1 \mu \mathrm{m}$ stacks; scale bars $2 \mu \mathrm{m}$; the data are representative of independent biological replicates and independent experiments. Source data are provided as a Source Data file

inhibition with BMH-21. Indeed, super-resolution confocal microscopy revealed that both VEX1 (Fig. 2a, Supplementary Fig. 3a) and VEX2 (Fig. 2b, Supplementary Fig. 3b) displayed significant levels of redistribution across the nucleus following inhibition of pol-I transcription, or inhibition of general transcription with actinomycin D (Supplementary Fig. 3c-f). Thus, recruitment of VEX1 and VEX2 to the VSG transcription compartment requires active pol-I transcription. VEX1 and VEX2 proteins remain within the nucleus when transcription is prevented, suggesting availability for retargeting once pol-I transcription reinitiates.

VEX2 depletion results in multi-VSG expression. VEX1 was initially identified from a high-throughput RNA interference (RNAi) screen for subtelomeric loss-of-silencing ${ }^{17}$. Another genome-wide RNAi screen indicated minimal fitness-cost following VEX1-knockdown, but a significant and major fitnesscost following VEX2-knockdown, specifically in bloodstreamform cells and not insect-stage cells ${ }^{26}$ (Supplementary Fig. 4a). This likely explains why only VEX1, and not VEX2, was identified in the loss-of-silencing screen, and indicates a bloodstream-form specific function for VEX2; VSG is not normally expressed in the insect-stage.

To explore the function of VEX2, we assembled three independent inducible RNAi knockdown strains with an active VSG-2 expression site (Fig. 3, Supplementary Fig. 4b). Knockdown was associated with a significant growth defect (Fig. 3a), but little perturbation of cell cycle distribution (Supplementary Fig. 4c). In these cells, normally silent VSG-6 was strongly derepressed after only $24 \mathrm{~h}$, as assessed by protein immunoblotting (Fig. 3b), immunofluorescence microscopy (Fig. 3c) and immunostaining followed by flow cytometry (Fig. 3d). Notably, abundance of the initially active VSG was also clearly reduced 
a

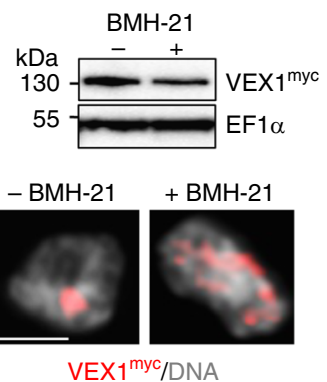

b
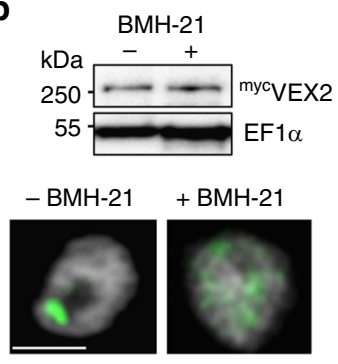

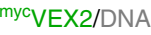
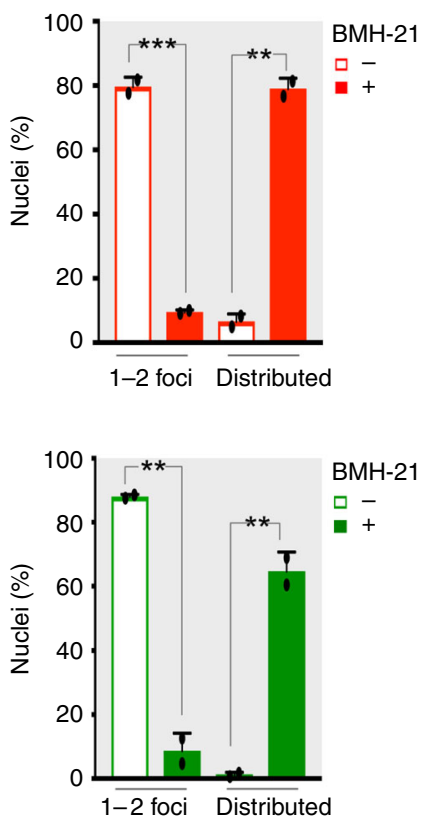

Fig. 2 VEX-complex compartmentalisation is transcription dependent. a, b Protein-blot and immunofluorescence analysis of VEX1myc (a) and mycVEX2 (b) before and after BMH-21 treatment (30 min at $1 \mu \mathrm{M})$. Proportions of nuclei displaying 1-2 major foci or distributed signals are indicated; the remaining cells displayed no detectable signal. Values are averages of two independent experiments ( $\geq 100$ nuclei each). Error bars, SD; ${ }^{\star \star} p<0.01 ;{ }^{\star \star \star} p<0.001$ (two-tailed paired Student's $t$ test). DNA was counter-stained with DAPl; the images correspond to maximal 3D projections of $0.1 \mu \mathrm{m}$ stacks; scale bars $2 \mu \mathrm{m}$; the data are representative of independent biological replicates and independent experiments. Source data are provided as a Source Data file after $48 \mathrm{~h}$ of VEX2-knockdown (Fig. 3b) and VSG-6 appeared to accumulate in intracellular compartments as well as at the surface (Fig. 3c, right-hand side). To survey additional VSGs, we used quantitative proteomics of proteins released from the cell-surface. The active VSG on wild-type cells, VSG-2, displayed a relative abundance of $99.9 \%$. Following VEX2-knockdown, VSG-2 remained the most abundant VSG, but VSG-6 and several additional ES-associated VSGs were derepressed (Fig. 3e, Supplementary Data 1 , sheet 3 ). Because antigenic variation occurs at low frequency, T. brucei clones are typically homogeneous and express a single VSG (Fig. 3c-e). Following VEX2-knockdown, however, $>90 \%$ of cells simultaneously expressed VSG-2, and VSG-6 at the cell surface as determined by microscopy (Fig. 3c) and flow cytometry (Fig. 3d). Taken together, these results indicate major disruption of allelic exclusion and the simultaneous expression of multiple VSGs on the surface of individual cells following VEX2-knockdown.

VEX2 coordinates allele-specific VSG-ES transcription. To further explore gene expression defects following VEX2-knockdown, we analysed the transcriptome, comparing three independent knockdown strains to three wild-type sub-clones (Supplementary Fig. 5, Supplementary Data 1, sheets 4-5). Since RNAi may not degrade the entire mRNA, we show efficient disruption of VEX2 transcript in Fig. 4a. Among approximately 7600 genes encoded in the genome, 166 displayed $>5$-fold and significantly $\left(p<10^{-2}\right)$ increased expression after $24 \mathrm{~h}$ knockdown (Fig. 4b-e), including every known pol-I transcribed protein-coding locus. There are 18 known silent, telomeric, pol-I promoter-associated VSGs in the genome; 13 in polycistronic expression sites and 5 in monocistronic expression sites, with the latter only normally activated in metacyclic cells in the insect salivary gland ${ }^{18}$. Total transcript abundance from this full set of 18 VSGs (Fig. 4b, c), and 130 silent expression-site associated genes (ESAGs) (Fig. 4d), was increased by 212 and 32 -fold, a

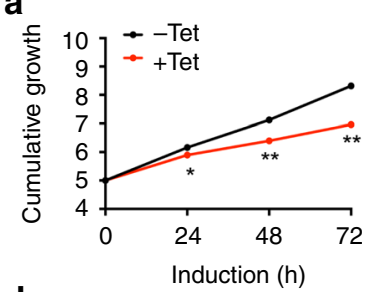

d
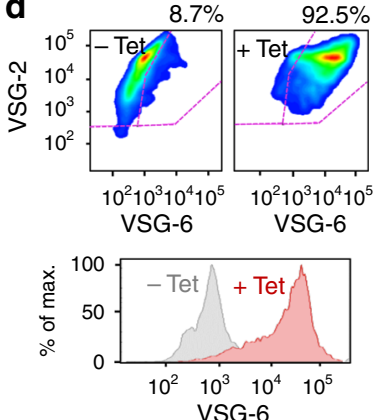

b

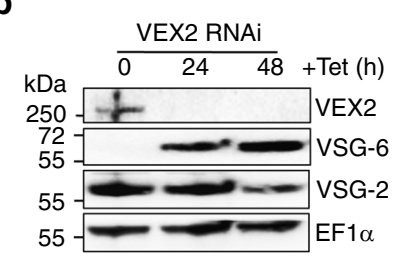

e

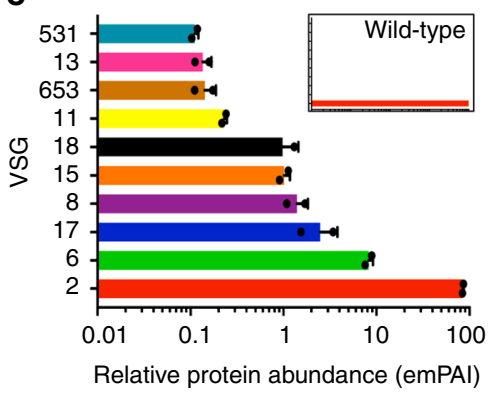

C

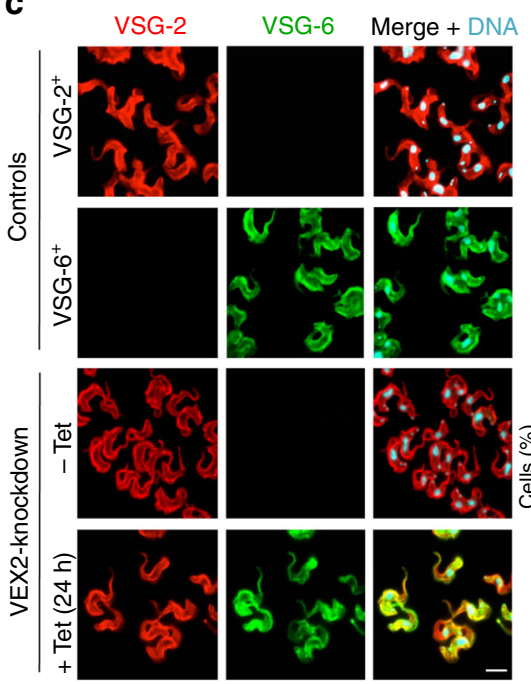

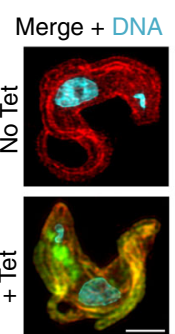

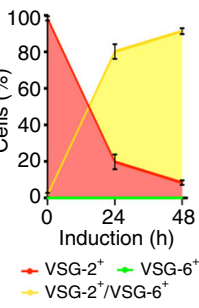

Fig. 3 VEX2 depletion results in multi-VSG expression. a Cumulative growth following tetracycline (Tet) induced VEX2-knockdown; ${ }^{\star} p<0.05 ;{ }^{\star \star} p<0.01$ (multiple $t$ tests). Protein blotting (b), immunofluorescence analysis (c), and flow cytometry (d), to assess VSG expression following VEX2-knockdown (24 h). EF1 $\alpha$, loading-control in (b); in c, DNA was counter-stained with DAPI and the graph depicts the rapid increase of dual VSG expressing cells following VEX2 knockdown by microscopy analysis. Scale bars, $5 \mu \mathrm{m}$ (left hand side) and $2 \mu \mathrm{m}$ (right hand side). Percentages in d indicate double-positive cells. e Quantitative mass spectrometry analysis of surface-VSGs following VEX2-knockdown ( $48 \mathrm{~h}$ ). The inset shows data for wild-type cells. emPAl, exponentially modified Protein Abundance Index. Error bars (not visible in $\mathbf{a}$ ), SD; data are averages from two (a/c/e) independent biological replicates and representative of independent experiments (a-d). Source data are provided as a Source Data file 


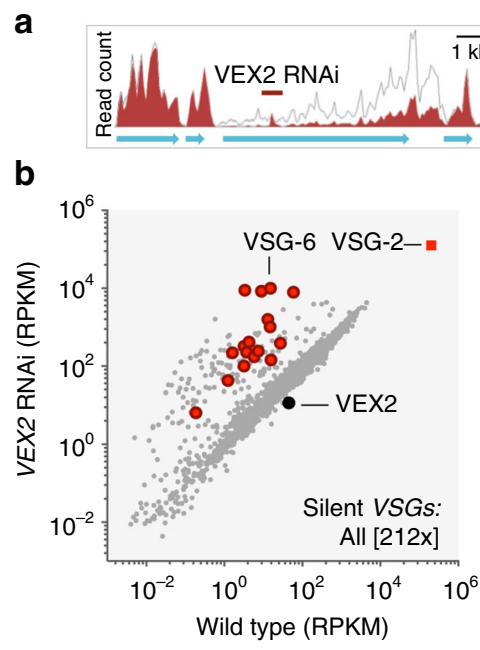

C

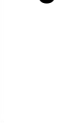

Silent VSGs: Dual promoter $[344 \times] \bullet$ Single promoter $[42 \times] \circ$
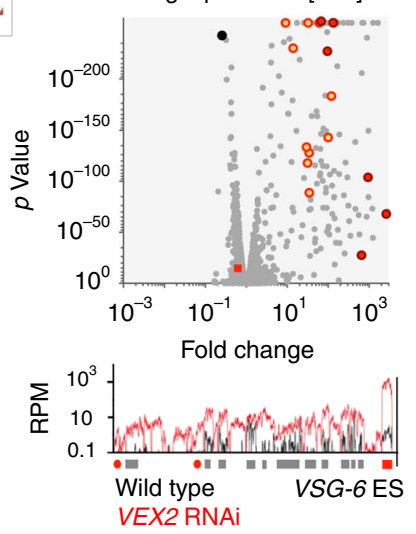

d

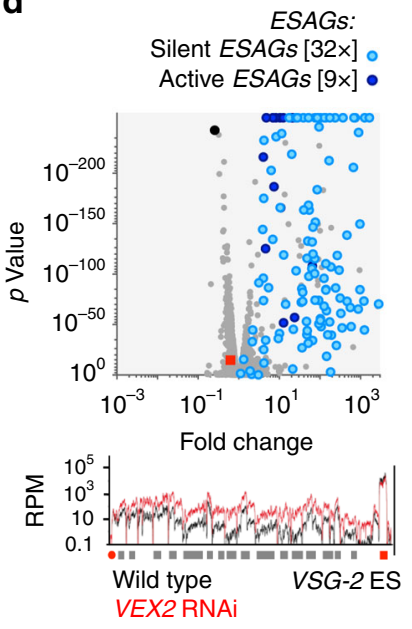

e

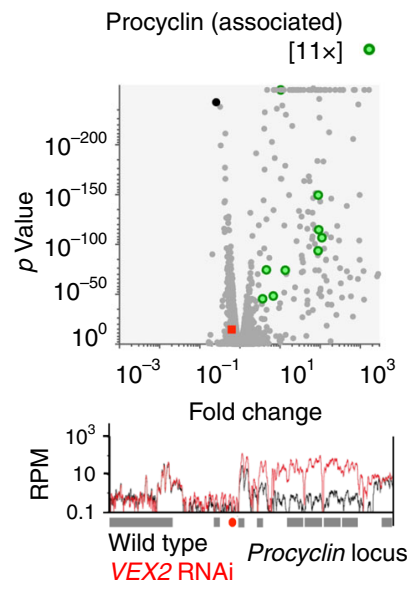

f
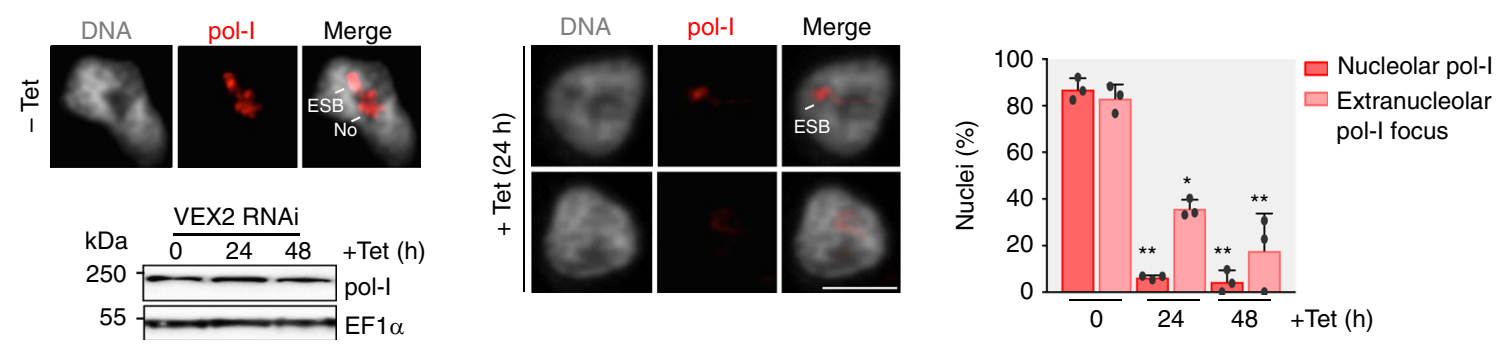

Fig. 4 VEX2 coordinates allele-specific VSG-ES transcription. a-e RNA-seq analysis following VEX2-knockdown (24h). Total number of reads at VEX2 locus in the parental population or following VEX2 RNAi (target fragment indicated) (a). b-e Values are averages from three independent RNAi strains relative to wild-type controls and numbers in brackets indicate increase in transcript abundance for each gene cohort. Red square, VSG-2; black circle, VEX2. Lower panels show transcript abundance at the silent VSG-6, active VSG-2 and procyclin loci, respectively. Red circles, promoters; red boxes, VSGs; grey boxes, other genes. RPKM/RPM, reads per (kilobase per) million. $\mathbf{f}$ Immunofluorescence and protein-blot analysis of pol-I expression and localisation following VEX2 RNAi. The bar graph indicates the proportion of nuclei $(n \geq 100)$ with nucleolar and/or extranucleolar pol-I signals; the remaining cells displayed no detectable signal. DNA was counter-stained with DAPI; the images correspond to maximal 3D projections of $0.1 \mu \mathrm{m}$ stacks; scale bars $2 \mu \mathrm{m}$. Error bar, SD; ${ }^{*} p<0.05 ;{ }^{\star \star} p<0.01$ (two-tailed paired Student's $t$ test); data are averages from three independent biological replicates and representative of independent experiments. Source data are provided as a Source Data file

respectively. Even the ESAGs at the active transcribed locus $(n=$ 18) were significantly $\left(p<10^{-50}\right)$ upregulated (9-fold, Fig. $4 \mathrm{~d}$ ), as were the non-telomeric, pol-I transcribed procyclin (associated) genes (Fig. 4e); procyclin genes produce abundant surface proteins that are normally expressed only in insect mid-gut stage cells. Highlighting the magnitude of the VEX2-knockdown phenotype, derepressed VSGs contributed $>24 \%$ of total VSG transcripts, compared to only $0.1 \%$ in control cells (Supplementary Data 1 , sheets 4-5); the super-abundant active VSG mRNA is $>200$-fold more abundant than the average mRNA encoding ribosomal proteins. Notably, several previously silent VSGs in ESs with dual promoters (VSG-6, 8, 15 and 17; see ref. ${ }^{7}$ ) were particularly derepressed and produced the four most abundant cellular mRNAs, after VSG-2; these VSGs were also readily detected at the cell-surface following VEX2-knockdown (Fig. 3e). The same VSG-ESs were activated at high rates during VSG switching ${ }^{27}$. Thus, an additional VSG-ES promoter likely facilitates the derepression or activation of a VSG-ES. We conclude that VEX2 coordinates VSG allelic exclusion and has a profound impact on differential VSG expression.

We did not anticipate increased expression of ESAGs at the active VSG-ES in response to VEX2-knockdown (Fig. 4d). However, it has long been known that ESAGs upstream of VSGs, despite co-transcription in the same polycistron, yield far less abundant transcripts, and no factor responsible for this differential control had been identified previously; VSG mRNA is $>140$-fold more abundant than the mean ESAG mRNA $^{18}$. Our transcriptome analysis now indicates post- or co-transcriptional suppression of these ESAGs mediated by VEX2. We speculate, based on sequence similarity of VEX2 to UPF1, that negative control by VEX2 may be related to nonsense-mediated mRNAdecay.

We next asked what impact VEX2-knockdown and VSG-ES derepression had on RNA pol-I localisation. Immunofluorescence microscopy revealed a remarkable loss of the nucleolar pol-I signal after only $24 \mathrm{~h}$ of knockdown and a substantial loss of detectable extranucleolar pol-I foci; protein blotting indicated that pol-I was still present following VEX2 knockdown (Fig. 4f). These results suggest that derepressed VSG-ESs deplete the nucleolus and the ESB of pol-I, redistributing the polymerase to multiple extranucleolar sites.

VEX-complex knockdown yields a severe exclusion defect. VEX1 knockdown yielded a moderate VSG derepression defect ${ }^{17}$ relative to the VEX2 knockdown phenotype (Figs. 3 and 4). We, therefore, knocked down both VEX1 and VEX2 simultaneously and carried out a similar analysis to that described above. VEX1-VEX2 double knockdown was associated with a very severe growth defect that was cytocidal after $72 \mathrm{~h}$ (Fig. 5a). In these cells, silent VSG-6 was again strongly derepressed, as assessed by protein immunoblotting; the active-VSG signal was 
a

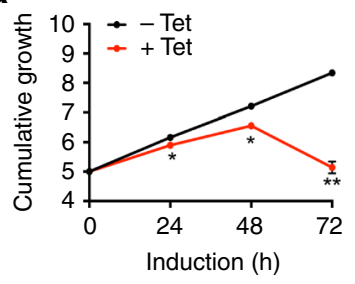

b

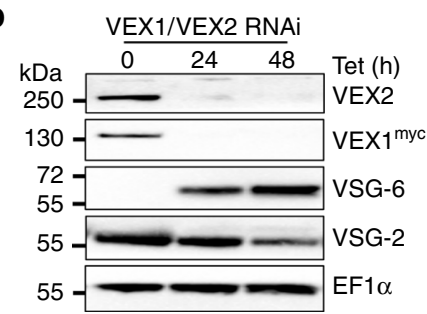

d

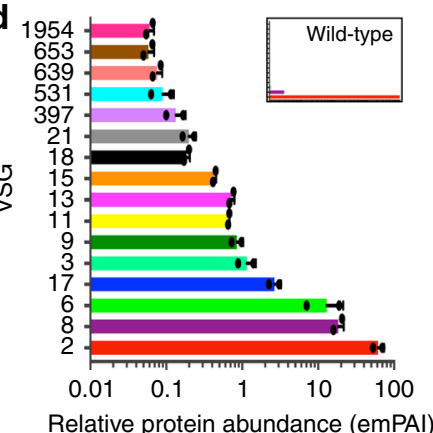

C

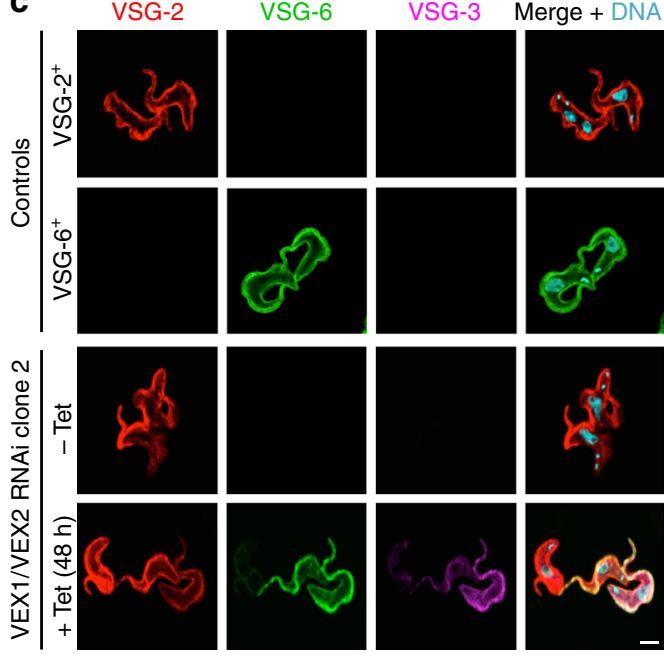

DNA/VSG-2/VSG-6
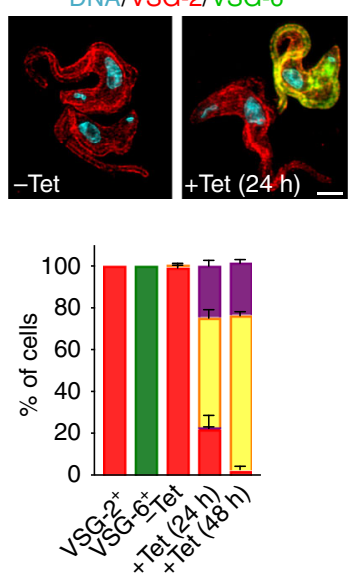

- VSG-2+ VSG-6 ${ }^{+}$VSG-3 ${ }^{+}$ $\square$ VSG-2+ VSG-6 $6^{+}$ ㅁ VSG-3+ - VSG-2

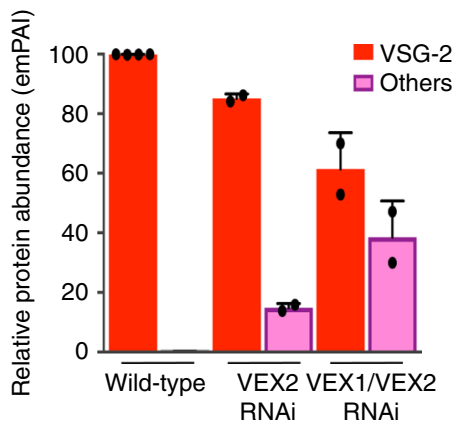

f
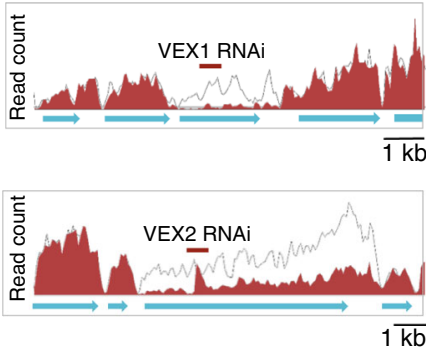

$1 \overline{\mathrm{kb}}$

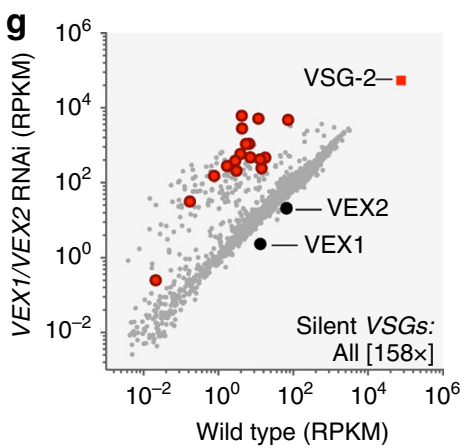

h

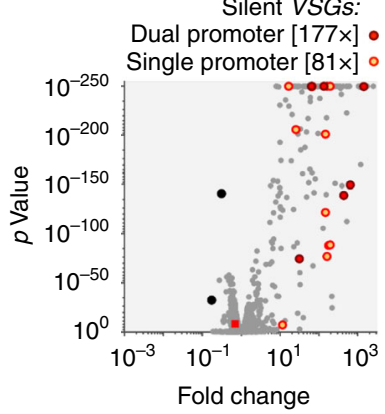

i

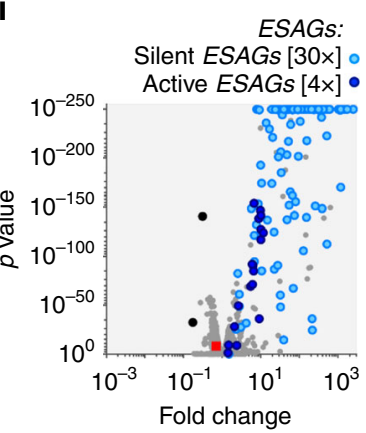

j

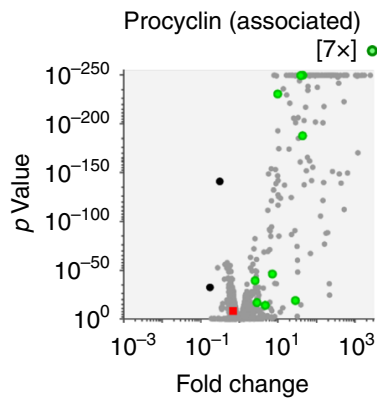

Fig. 5 VEX-complex knockdown yields a severe exclusion defect. a Cumulative growth following tetracycline (Tet) induced VEX1-VEX2 double knockdown; ${ }^{\star} p<0.05 ;{ }^{\star \star} p<0.01$ (multiple $t$ tests). Protein blotting (b), and immunofluorescence analysis (c), to assess VSG expression following VEX1-VEX2 double knockdown (24 h). Other details as in Fig. 3a-c. In c, an additional silent VSG, VSG-3, was analysed. d Quantitative mass spectrometry analysis of surfaceVSGs following VEX1-VEX2 double knockdown (48 h). The inset shows data for wild-type cells. e Relative abundance of surface-VSGs following VEX2 or VEX1/VEX2-knockdown (48 h). emPAl, exponentially modified Protein Abundance Index (d, e). Data are averages from two independent biological replicates (a, c-e) and representative of independent experiments (a-c). Error bars, SD. f-j RNA-seq analysis following VEX1-VEX2 double knockdown $(24 \mathrm{~h})$. Total number of reads across VEX1 and VEX2 loci in the parental population or following knockdown (f). In $\mathbf{g}$-j specific cohorts of genes are highlighted and values are averages from three independent RNAi strains relative to wild-type controls. Numbers in square brackets indicate increase in total transcript abundance for each category; RPKM, reads per kilobase per million. Source data are provided as a Source Data file

once again clearly reduced after $48 \mathrm{~h}$ of knockdown (Fig. 5b). Following $48 \mathrm{~h}$ of VEX1-VEX2 double knockdown, immunofluorescence microscopy revealed that almost all cells expressed both VSG-2 and VSG-6, while $>20 \%$ of cells simultaneously expressed VSG-2, VSG-6 and VSG-3 (Fig. 5c); VSG-13 was also expressed by $>20 \%$ of these cells (Supplementary Fig. 6a). We further monitored VEX1-knockdown cells over an extended period, during which cells remain viable ${ }^{17}$. This revealed $>25 \%$ of the population expressing both VSG-2 and VSG- 6 throughout the time-course, with no evidence for switching to VSG-6 expression (Supplementary Fig. 6b). Thus, VEX-complex knockdown disrupts allelic exclusion and does not simply increase the VSG switching rate. Quantitative proteomic analysis following VEX1-VEX2 knockdown revealed derepression of 15 pol-I promoter-associated VSGs (Fig. 5d, Supplementary Data 1, sheet 3). A comparison of relative VSG expression revealed that derepressed VSGs contributed approx. $40 \%$ of total VSG following VEX1-VEX2 knockdown (Fig. 5e, Supplementary Data 1, sheet 
a

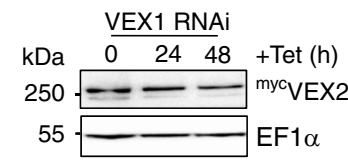

VSG-2 VSG-6 DNA
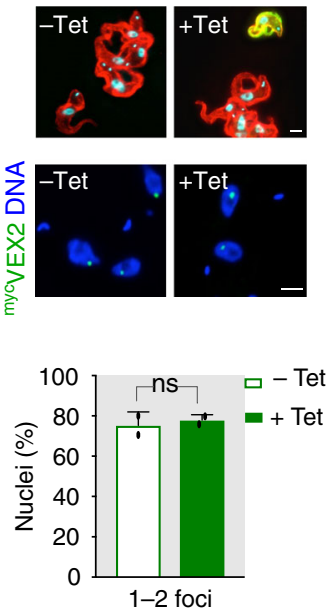

C

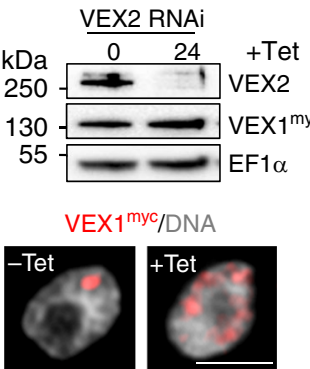

b

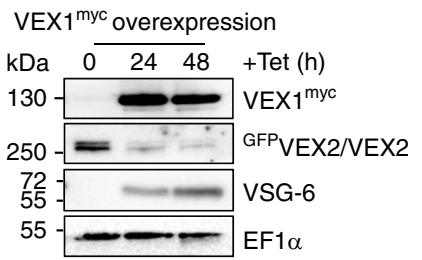

VSG-2 VSG-6 DNA
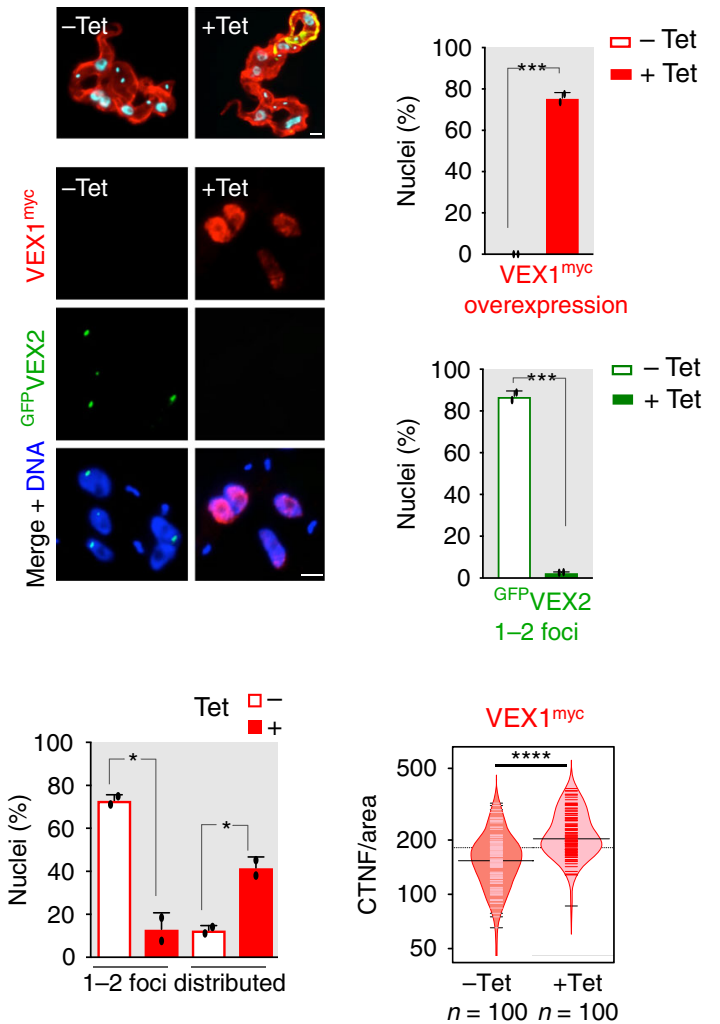

Fig. 6 VEX-complex interactions impact abundance and location. a, b Protein-blot and immunofluorescence analysis following Tet-induced VEX 1 knockdown and Tet-induced mycVEX1 overexpression, respectively $(48 \mathrm{~h})$. In the upper immunofluorescence panels, cells were stained with $\alpha$-VSG-2 and $\alpha$-VSG-6 to confirm the dual VSG expression phenotypes reported previously ${ }^{17}$. In the lower panels, cells were stained with $\alpha$-myc for VEX2 localisation (a) or both $\alpha$-myc and $\alpha$-GFP for VEX1 and VEX2 localisation, respectively (b). The bar graphs indicate the proportion of $\geq 300$ nuclei with 1-2 myc VEX2 foci following $48 \mathrm{~h}$ of VEX1 knockdown (a) or with 1-2 GFPVEX2 foci following $48 \mathrm{~h}$ of VEX1 overexpression (b). The proportion of nuclei displaying a distributed VEX1 signal (due to overexpression) is also shown in $\mathbf{b}$; the remaining cells displayed no detectable signal. c Protein-blot and immunofluorescence analysis of VEX1Myc following Tet-induced VEX2 knockdown (24 h). The bar graph indicates the proportion of nuclei with 1-2 VEX $1^{\mathrm{myc}}$ foci or distributed signal, the remaining cells displayed no detectable signal. The bean-plot shows signal intensity in $\geq 100$ nuclei with 1-2 major VEX1myc foci ( - Tet) or nuclei with a distributed signal (+Tet $24 \mathrm{~h}$ ) following VEX2 knockdown. Black lines show the medians; pink/red lines represent individual data points; polygons represent the estimated density of the data. CTNF corrected total nuclear fluorescence, normalised to the nuclear area. EF1 $\alpha$ was used as a loading-control. DNA was counter-stained with DAPI; scale bars $2 \mu \mathrm{m}$. The images in (c) correspond to maximal 3D projections of $0.1 \mu \mathrm{m}$ stacks. For bar graphs, data representative of independent experiments and averages from two independent biological replicates; error bars, SD. ns, non-significant; ${ }^{\star} p<0.05$; ${ }^{\star \star \star} p<$ $0.001 ;{ }^{\star \star \star \star} p<0.0001$ (two-tailed paired and unpaired Student's $t$ tests applied to bar graphs and bean plot, respectively). Source data are provided as a Source Data file

3) compared to only approximately $10 \%$ of total VSG following VEX2 knockdown. As for VEX2-knockdown (Fig. 4f), double knockdown was associated with loss of both the nucleolar pol-I signal and detectable extranucleolar pol-I foci (Supplementary Fig. 6c).

Transcriptome analysis following VEX1-VEX2 knockdown (Fig. 5f, Supplementary Fig. 7, Supplementary Data 1, sheets 4-5) revealed a similar pattern of derepression as observed above for VEX2 knockdown alone (Fig. 5g-j). Again, all known pol-I transcribed protein-coding loci were derepressed, and previously silent VSGs linked to dual VSG-ES promoters produced the four most abundant cellular mRNAs, after VSG-2 (Fig. 5g). These data demonstrate that the VEX-complex coordinates VSG allelic exclusion. The allelic exclusion system collapses following depletion of the VEX-complex, yielding multi-VSG expression, highly heterogeneous VSG coats and cell-death.

VEX-complex interactions impact abundance and location. We next asked what impact VEX1 and VEX2 have on each other. VEX1-knockdown had no detectable impact on VEX2 expression or focal localisation (Fig. 6a). In contrast, VEX1 over-expression substantially diminished VEX2 abundance; VEX1 was highly overexpressed in most nuclei $(\sim 80 \%)$ and VEX2 foci were no longer detectable by immunofluorescence (Fig. 6b). These data 

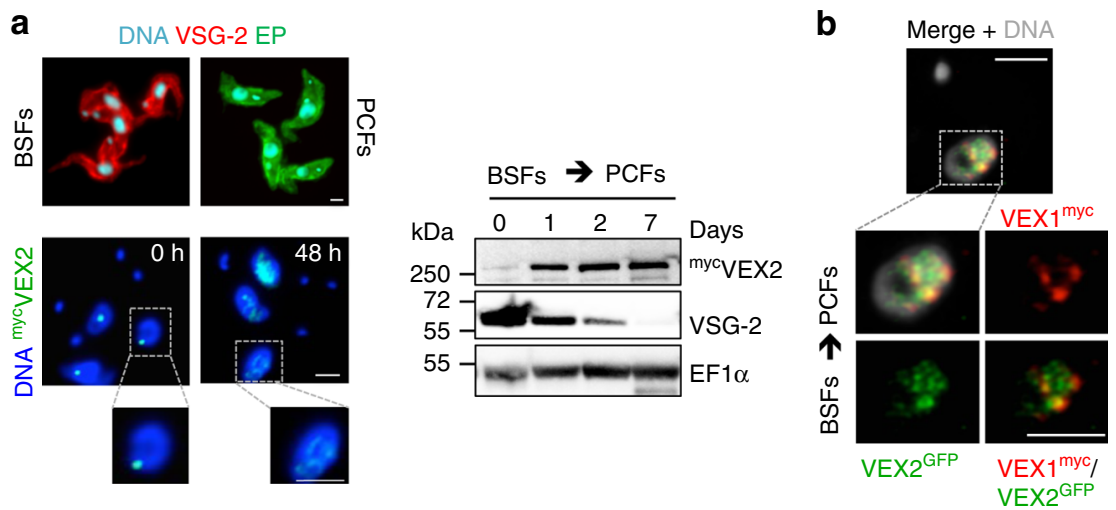

Fig. 7 VEX-complex compartmentalisation is stage-specific. a Immunofluorescence and protein-blot analysis of mycVEX2 localisation and expression, respectively, following differentiation of bloodstream forms (BSFs, $\mathrm{O}$ ) into procyclic forms (PCFs). As immunofluorescence control, the cells were stained for the major surface glycoprotein for each developmental stage; VSG in BSFs and EP-procyclin in PCFs. For protein blotting, EF1 $\alpha$ was used as a loadingcontrol. b GFPVEX2/VEX1myc colocalisation studies in $48 \mathrm{~h}$ differentiated BSFs. In $\mathbf{b}$, these images correspond to maximal $3 \mathrm{D}$ projections of $0.1 \mu \mathrm{m}$ stacks. DNA was counter-stained with DAPl; scale bars $2 \mu \mathrm{m}$. Data representative of independent experiments. Source data are provided as a Source Data file

likely explain the previously reported more severe VSG derepression phenotype following VEX1 over-expression compared with knockdown ${ }^{17}$ and also indicate that VEX1 can limit VEX2 abundance; this latter feature may be important for preventing activation of a second VSG. We also tested the impact of VEX2knockdown on VEX1 localisation and found that VEX1 was redistributed across multiple sub-nuclear puncta following loss of VEX2 (Fig. 6c). Thus, VEX1 association with the VSG transcription compartment is VEX2-dependent.

VEX-complex compartmentalisation is stage-specific. Insectstage $T$. brucei cells neither transcribe VSG nor exhibit allelic exclusion. Since we show above that VEX-complex association with the VSG transcription compartment requires pol-I transcription, we asked whether VEX2 association with this compartment is absent in insect-stage cells. Like VEX1 ${ }^{17}$, VEX2 was indeed redistributed through the nuclear compartment during differentiation (Fig. 7a). Protein blotting revealed substantial upregulation of VEX2 as the VSG signal diminished (Fig. 7a) and these differentiated cells displayed multiple VEX2-foci, some of which were coincident with VEX1-foci (Fig. 7b). Redistributed VEX2 was similarly observed in long-term established insectstage cells. Thus, both VEX1 and VEX2 associate with the VSG transcription compartment in a pol-I transcription-dependent and life cycle stage-specific manner.

Colocalisation of CAF-1 and the VEX-complex during $S$ phase. We next considered the interaction between the VEX-complex and CAF-1; all three components of this conserved chromatin chaperone were co-immunoprecipitated with VEX1 (see Fig. 1b). DNA replication presents both an opportunity to retain or reset epigenetic states. We, therefore, asked if CAF-1 plays a role in the inheritance of the VEX-complex-dependent epigenetic state during DNA replication; the active VSG-ES is replicated early during S-phase while the silent VSG-ESs are replicated later ${ }^{28}$. Significantly, S-phase specific VSG-ES derepression was previously reported following CAF-1b knockdown ${ }^{15}$. By analysing the localisation of a tagged CAF- $1 \mathrm{~b}$ subunit, either CAF- $1 \mathrm{~b}^{\mathrm{GFP}}$ or CAF- $1 b^{\text {myc}}$, we observed major CAF-1b foci, despite an overall punctate nuclear distribution. Notably, these foci co-localised with the sub-nuclear compartment defined by both VEX1 (Fig. 8a, Supplementary Movie 2) and VEX2 (Fig. 8b) and coincident signals were significantly enriched in S-phase cells (Fig. 8a, b); the interaction between the VEX-complex and CAF-1 was also confirmed by immunoprecipitation followed by protein blotting (Fig. 8c).

VEX-complex reassembly and inheritance requires CAF-1. By analysing phenotypes associated with CAF-1b knockdown, we confirmed two further specific predictions in support of CAF-1 dependent inheritance of the VEX-complex. First, VEX1 distributed across multiple sub-nuclear puncta following CAF-1b knockdown (12 h, Fig. 9a); while VEX2 notably remained primarily compartmentalised in a single focus in each nucleus under the same conditions (Fig. 9b). Indeed, we observed more intense VEX2 foci following CAF-1b knockdown, and protein blotting confirmed that VEX2 abundance is specifically increased when CAF-1b is depleted (Fig. 9c, Supplementary Fig. 8a, b). Thus, CAF-1 limits VEX2 abundance, an effect that may be enhanced by excess VEX1 (see above). Second, following CAF-1b knockdown (see Fig. 9d), transcriptome analysis revealed specific derepression of VSGs and other pol-I transcribed protein-coding genes (Fig. 9e-i, Supplementary Fig. 8c, d, Supplementary Data 1, sheets 4-5).

Following CAF-1b knockdown for $24 \mathrm{~h}$ (see Supplementary Fig. 8c), 115 genes displayed $>5$-fold and significantly $\left(p<10^{-2}\right)$ increased expression, $>80 \%$ of which were also significantly increased following VEX2 knockdown (Supplementary Data 1, sheets 4,5$)$. In this case, we observed a more pronounced increase in total transcript abundance for promoter-proximal VSGs (89fold, $n=5$, Fig. 9g) relative to promoter-distal VSGs (9-fold, $n=$ 5, Fig. 9g). Similarly, we observed a more pronounced increase in total transcript abundance for promoter-proximal ESAGs (48-fold, $n=34$, Fig. 9h) relative to promoter-distal ESAGs (4-fold, $n=96$, Fig. 9h). Procyclin (associated) genes were also derepressed (6-fold, Fig. 9i), while ESAGs at the active locus displayed significantly $\left(p<10^{-4}\right)$ reduced expression $(44 \pm 19 \%$, $n=12$; Fig. 9h, Supplementary Data 1, sheets $4-5)$, consistent with increased VEX2 abundance and enhanced VEX2-mediated negative control at this locus (see above). Thus, CAF-1 knockdown has a major impact on pol-I transcribed protein-coding loci; promoter-proximal genes may be particularly susceptible to derepression due to a genome-wide histone chaperone defect.

A model for VSG allelic exclusion by the VEX-complex. Finally, combining RNA-seq data, we compared the relative contributions of VEX1, VEX2 and CAF1-b to VSG silencing (Fig. 10a) and also calculated relative contributions to an allelic exclusion index; the 
a

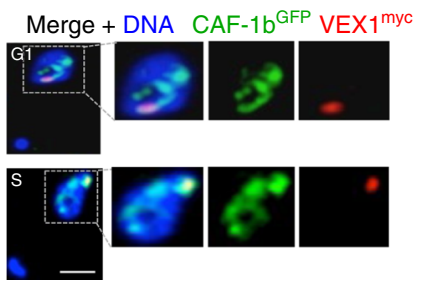

b

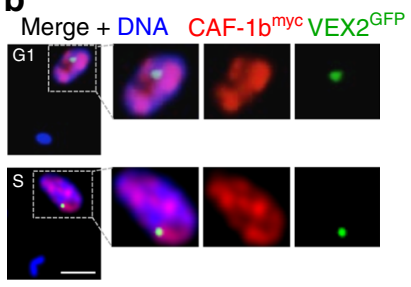

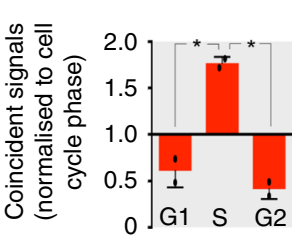

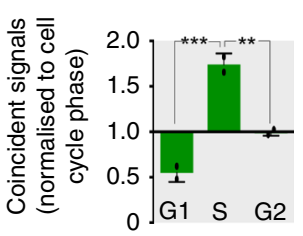

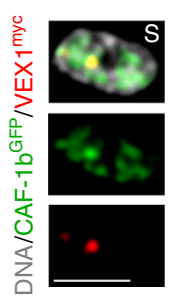

C
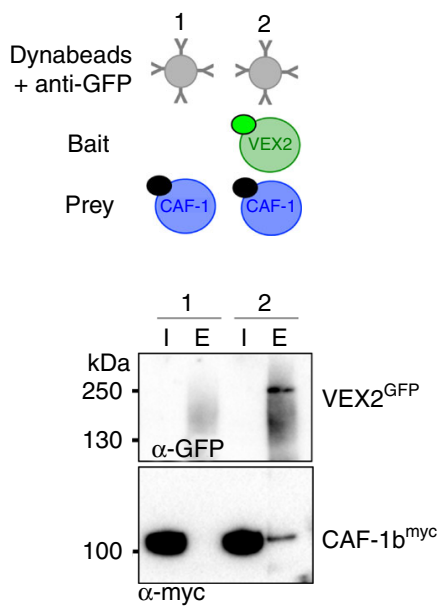

Fig. 8 Colocalisation of CAF-1 and the VEX-complex during S phase. $\mathbf{a}, \mathbf{b}$ Immunofluorescence-based colocalisation studies of VEX1myc/CAF-16 GFP and VEX2 $2^{\text {GFP }} / \mathrm{CAF}-1 \mathrm{~b}^{\mathrm{myc}}$. Representative images from G1 and S phase cells are shown. Nuclei $(n \geq 70)$ were scored for CAF-1b puncta coincident with VEX1myc/VEX2 ${ }^{\text {GFP }}$ foci. DNA was counter-stained with DAPI; scale bars, $2 \mu \mathrm{m}$. Error bars, SD; ${ }^{\star} p<0.05 ;{ }^{\star \star} p<0.01 ;{ }^{\star \star \star} p<0.001$ (one-way ANOVA test). Values are averages from two independent biological replicates and are representative of independent experiments. The images on the right-hand side correspond to maximal 3D projections of $0.1 \mu \mathrm{m}$ stacks. c Co-immunoprecipitation of VEX2 GFP/CAF-16myc followed by protein-blot analysis. Green circle, GFP; black circles, myc; I, input; E, elution. Source data are provided as a Source Data file

a

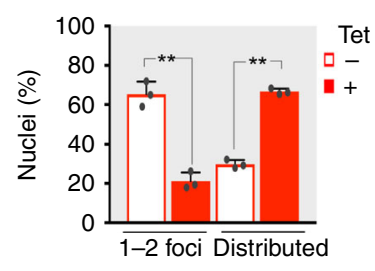

C

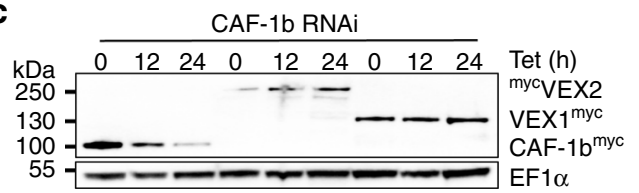

f

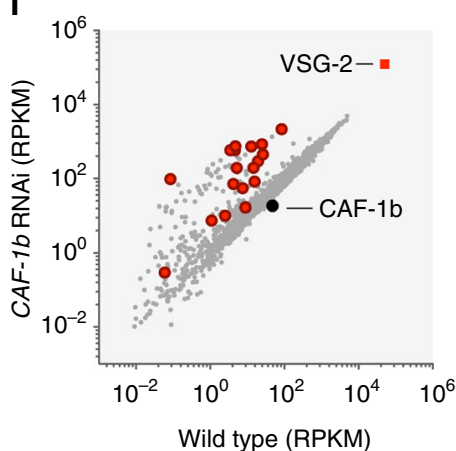

g

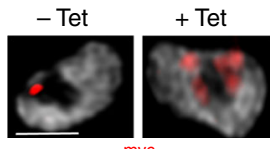

VEX1 ${ }^{\text {myc } / D N A}$

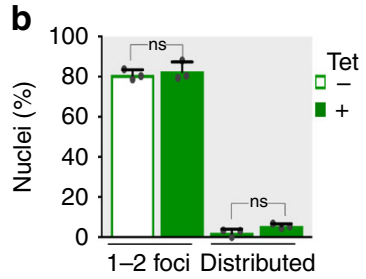

d

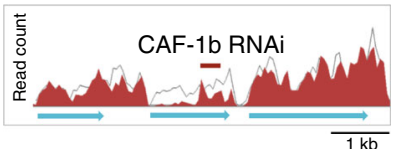

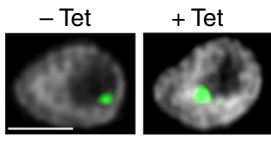

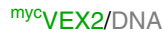

e

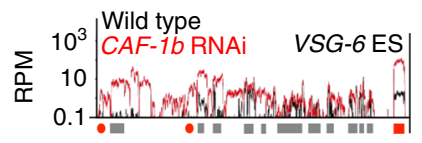

h

Silent VSGs: Dual promoter [26x]
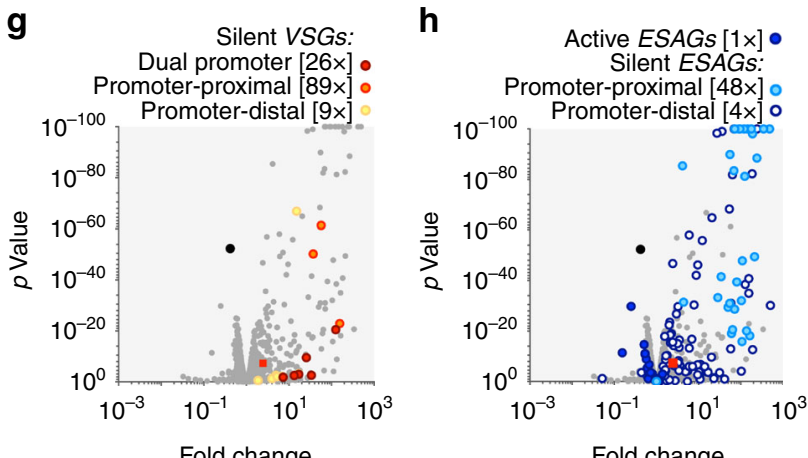

i

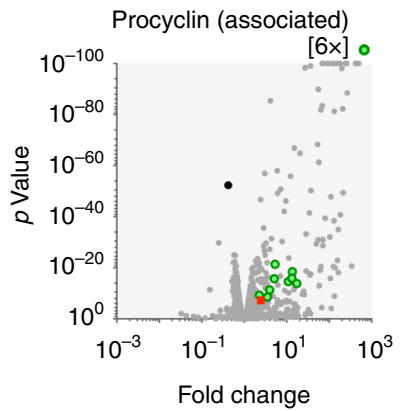

Fig. 9 VEX-complex reassembly and inheritance requires CAF-1. VEX1myc (a), but not mycVEX2 (b), is distributed following tetracycline (Tet) induced CAF$1 \mathrm{~b}$ knockdown $(12 \mathrm{~h}$ ), as assessed by immunofluorescence analysis. DNA was counter-stained with DAPl; scale bars, $2 \mu \mathrm{m}$. The images correspond to maximal 3D projections of $0.1 \mu \mathrm{m}$ stacks. Error bars, SD; ns, not significant; ${ }^{\star \star} p<0.01$ (two-tailed paired Student's $t$ test). Values are averages from three independent biological replicates and are representative of independent experiments (the remaining cells displayed no detectable signal). $\mathbf{c}$ Protein-blot analysis of CAF-1b myc, myc VEX2 and VEX1myc expression following CAF-1b knockdown. EF1 $\alpha$ was used as a loading-control. d-i RNA-seq analysis following CAF-1b knockdown $(24 \mathrm{~h})$. d Total number of reads across CAF-1b locus in the parental population or following CAF-1b RNAi. e-i Values are averages from two independent RNAi strains relative to wild-type controls. Panel e shows transcript abundance at the silent VSG-6 ES. Red circles, promoters; red boxes, VSGs; grey boxes, other genes. RPKM/RPM, reads per (kilobase per) million (e-i). Panels f-i highlight different gene cohorts and numbers in square brackets indicate increase/decrease in total transcript abundance for each category; red square, VSG-2; black circle, CAF-1b. Promoter-proximal VSGs and ESAGs $(6,7$ and 10) are within $5 \mathrm{kbp}$ of a promoter, distal VSGs are $46+/-4 \mathrm{kbp}$ from the promoter. Source data are provided as a Source Data file 
a

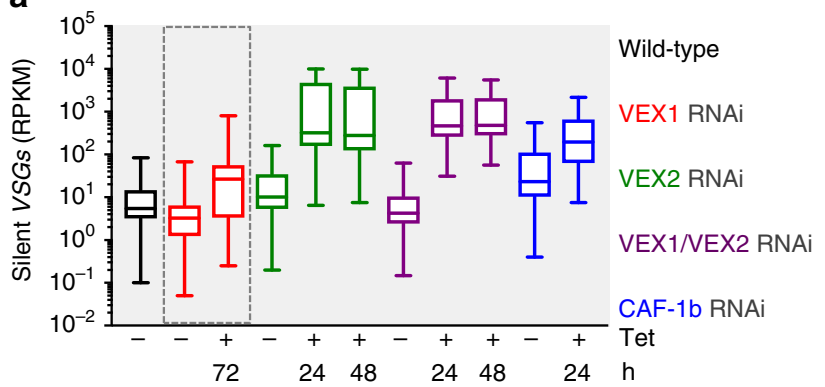

C

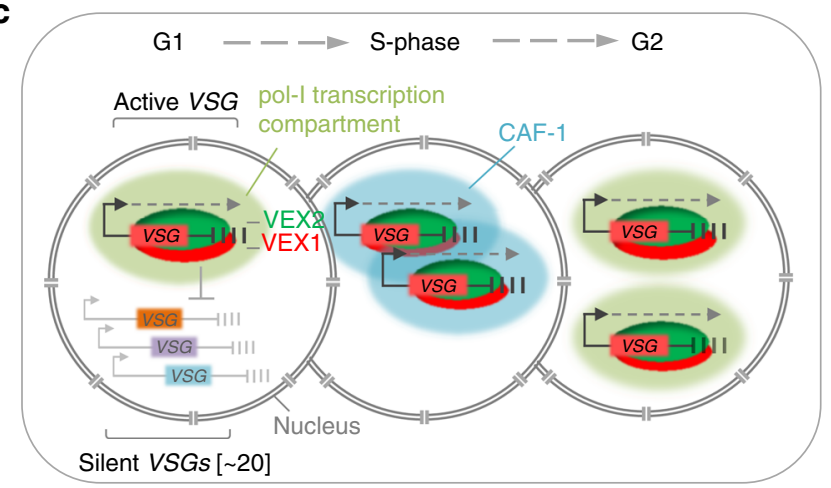

b
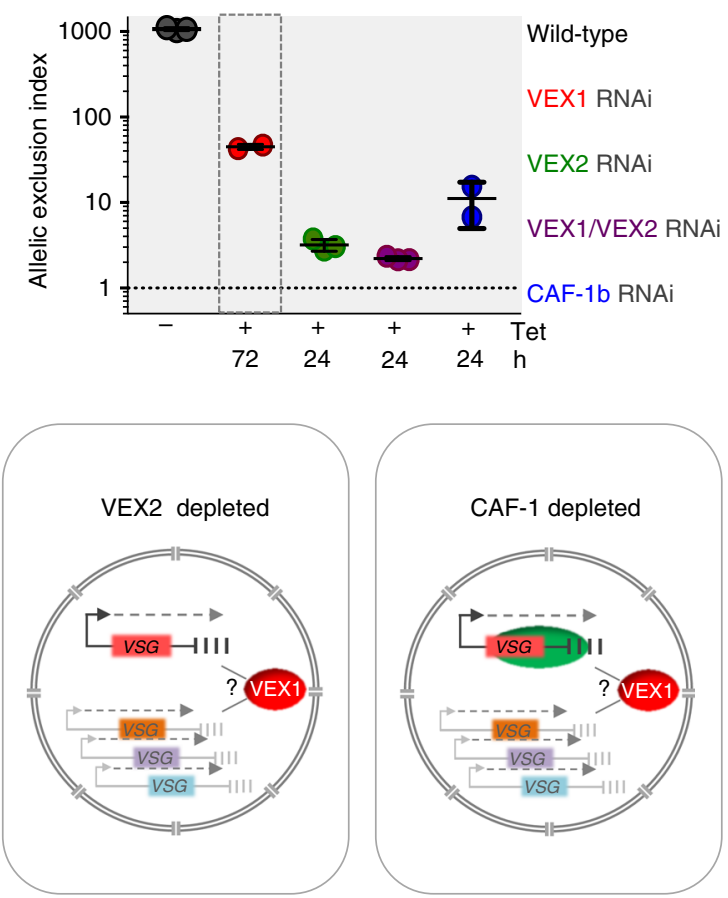

Fig. 10 A model for VSG allelic exclusion by the VEX-complex. a, b Comparative analysis of VSG-transcripts from wild-type T. brucei or following VEX1 (data from Glover et al., 2016; dashed boxes), VEX2, VEX1/VEX2 and CAF-1b knockdown (data from current study). a The box-plot depicts the sum of silent VSG expression; whiskers extend from the minimal to the maximal value, other details as in Fig. 1a. $\mathbf{b}$ The allelic exclusion index represents active VSG-2 expression divided by the expression of all other expression site associated VSGs combined. Error bars, SD. c A model for heritable exclusion by the VEX-complex and CAF-1. Left-hand panel: VSG-ES transcription drives competition for a limited pool of the VEX-complex. Once associated with chromatin, the complex promotes transcription and transmits a silencing signal, excluding other VSG-ESs, ultimately allowing only one VSG-ES to recruit sufficient polI and to form a pol-I transcription compartment. CAF-1 dependent compartmentalisation of the complex provides allele-specific epigenetic inheritance. Middle and right-hand panels: VEX2 or CAF-1 depletion disrupts the VEX-complex associated transcription compartment and the silencing signal; it remains unclear how VEX1 interacts with VSG-ESs under these circumstances. Bent arrows and dashed arrows indicate VSG-ES promoters and transcription, respectively. Source data are provided as a Source Data file

ratio of reads from the active VSG relative to all 18 silent expression-site associated VSGs (Fig. 10b). VEX2 clearly makes the major contribution to VSG silencing (Fig. 10a, b), establishing a 1000-fold expression differential in wild-type cells, which, following VEX2 or VEX-complex depletion, is diminished to a 2-4fold differential (Fig. 10b). As illustrated by our model (Fig. 10c), we conclude that the VEX-complex associates with the active $V S G$ in a pol-I transcription-dependent manner and thereby coordinates nuclear pol-I compartments and VSG exclusion. VEX1 compartmentalisation is VEX2-dependent, while retention during DNA replication is also CAF-1 dependent.

\section{Discussion}

An improved understanding of immune evasion by pathogens has profound importance to health and disease. Despite intense study, mechanisms underlying stochastic activation of one allele and the heritable exclusion of all others remain to be fully defined. This has been the case for antigenic variation in the parasites that cause malaria ${ }^{1}$, giardiasis ${ }^{2}$ and African trypanosomiasis; ${ }^{3}$ and also in mammals where olfactory receptor allelic exclusion underpins odour detection ${ }^{4}$ and where immunoglobulin and receptor allelic exclusion underpin specificity in B and T cells ${ }^{5}$.

Here, we describe an association between a bipartite VSG exclusion (VEX)-complex and the active VSG transcription sub-compartment in T. brucei. The complex, which is compartmentalised due to active VSG-ES transcription, mediates transcriptional silencing of all other VSG alleles (Fig. 10c). In addition, VEX1 sequestration requires VEX2, and also CAF-1 during DNA replication. Transcription-dependent sequestration, and a major allelic exclusion defect when the VEX-complex is depleted are consistent with a sequestration-transcription based positive-feedback mechanism, favoring sequestration of the VEX-complex at the transcribed VSG compartment. This may be important for maintaining, and we speculate, also establishing, allelic exclusion. CAF-1 dependence indicates that inheritance of exclusion requires VEX1 reloading during, or soon after, DNA replication. Notably, the VEX-complex is also present in insect-stage cells, suggesting availability for retargeting once VSG transcription reinitiates in parasites in the insect salivary gland.

Several observations suggest potentially shared mechanisms with allelic exclusion in mammals. The VEX2-related UPF1 orthologue in humans (Rent1) influences early nuclear events in mRNA biogenesis, including splicing ${ }^{29}$. Mammalian Rent1 is enriched at telomeres and negatively controls the association between telomeric repeat-containing RNA (TERRA) transcripts and chromatin at these sites; it has been proposed that these transcripts promote heterochromatin assembly, similar to Xist RNA promotion of $\mathrm{X}$-chromosome inactivation ${ }^{30}$. In addition, murine UPF1 is required for X-inactivation and formation of Xist RNA domains, a function involving Xist splicing control ${ }^{31}$. Taken together, these findings suggest a conserved role for this helicase family in regulating lncRNA-chromatin associations, and possibly in gene expression choices. The enrichment of VEX1 and VEX2 in association with the active VSG-ES is also reminiscent of 
nuclear sub-structures in other eukaryotic cells, such as PML bodies and nuclear speckles ${ }^{32,33}$.

It remains unclear how the activity state of a gene is reproduced on daughter chromatids following DNA replication. The conserved hetero-trimeric histone $\mathrm{H} 3-\mathrm{H} 4$ specific chaperone, CAF-1, participates in chromatin assembly directly behind the passing DNA polymerase during DNA replication, integrating DNA synthesis with conservative reassembly of chromatin in eukaryotes 24,34 . This involves reincorporating the majority of maternal histones within $400 \mathrm{bp}$ of their pre-replication locus ${ }^{35}$ and locus-specific bookmarking by epigenetic regulatory complexes to maintain transcriptional programs ${ }^{36,37}$. Mammalian CAF-1 sustains somatic cell types ${ }^{38}$ and epigenetic memory by interacting with heterochromatin protein $1 \alpha$, a histone $\mathrm{H} 3$ methyltransferase and KRAB-ZFP-associated protein 1; this complex is also associated with sub-nuclear foci ${ }^{39}$. Human CAF-1 can also activate transcription independent of its histone deposition function ${ }^{40}$ and the conserved CAF-1 subunit-associated dREAM complex plays a role in specifying and maintaining olfactory receptor gene expression in Drosophila ${ }^{41}$. Thus, in addition to housekeeping roles in chromatin assembly, several lines of evidence suggest an evolutionary conserved role for CAF1 in sustaining site-specific molecular memories of epigenetic states. Our findings indicate that this can involve CAF-1 dependent and locus-specific compartmentalisation of non-histone, chromatin-associated regulators as epigenetic marks. Consistent with this view, a cohesin-dependent delay in active VSG sister chromatid separation facilitates epigenetic inheritance ${ }^{42}$. Thus, premature segregation of sister chromatids following cohesin depletion may yield free VEX1 and present opportunities for activation of other VSG-ESs.

VEX2 makes the greatest contribution to differential VSG expression, as assessed at mRNA and protein levels, while loss of both VEX1 and VEX2 leads to collapse of the exclusion system. Transcription sustains VEX-complex assembly at the active locus and this sequestration through transcription may also drive the establishment of a single active site. The VEX-complex may also be self-limiting, in the sense that CAF-1 and VEX1 can negatively control VEX2 expression; this may be important to minimise activation at other sites and hence secure selectivity. We previously demonstrated homology-dependent silencing by VEX ${ }^{17}$ and suspect that communication among VSG alleles requires a trans-acting RNA component. Although the current findings demonstrate robust negative control of silent VSGs by the VEXcomplex, a more detailed dissection of the proposed positive and negative controls, and their coordination to achieve allelic exclusion, will require further study.

In summary, the crosstalk among VSG-ESs and inheritance of allelic exclusion in trypanosomes requires transcription, the VEXcomplex and CAF-1 dependent partitioning of VEX1. These factors collectively sustain a specific assembly in association with the active VSG, resulting in exclusion of all other VSG alleles. CAF-1 links the VEX-complex to the active site to produce a molecular memory. Our findings reveal the factors underpinning a winner-takes-all paradigm for the establishment, maintenance and inheritance of nuclear bodies and allele-specific epigenetic states. To our knowledge, this is the first characterisation of a protein complex directly responsible for single gene choice in an allelic exclusion system.

\footnotetext{
Methods

T. brucei growth and manipulation. Bloodstream-form T. brucei, Lister 427 and $2 \mathrm{~T} 1$ cells $^{43}$, both wild-type with respect to VEX1, VEX2 and CAF-1 subunits, were grown in HMI-11 medium and genetically manipulated using electroporation ${ }^{44}$; cytomix was used for all transfections. Puromycin, phleomycin, hygromycin and blasticidin were used at $2,2,2.5$ and $10 \mu \mathrm{g} \mathrm{ml}^{-1}$ for selection of recombinant
}

clones; and at $1,1,1$ and $2 \mu \mathrm{g} \mathrm{ml}^{-1}$ for maintaining those clones, respectively. Cumulative growth curves were generated from cultures seeded at $10^{5}$ cells $\mathrm{ml}^{-1}$ counted on a haemocytometer and diluted back to $10^{5}$ cells $\mathrm{ml}^{-1}$ as necessary. For differentiation of bloodstream form to procyclic form, $2 \times 10^{7}$ cells were resuspended in DTM medium, $2.5 \mathrm{mg} \mathrm{ml}^{-1}$ of haemin, $300 \mathrm{mM}$ cis-aconitate and incubated at $27^{\circ} \mathrm{C}$. Established procyclic-form T. brucei, Lister 427 cells were grown in SDM-79 at $27^{\circ} \mathrm{C}$ and genetically manipulated using electroporation as above. Blasticidin was used at 10 and $2 \mu \mathrm{g} \mathrm{ml}^{-1}$ for selection and maintenance, respectively.

Plasmids. For RNAi, primers were selected from ORF sequences using CLC Viewer v. 7.8 and BLAST analysis to minimise potential off-target effects. A specific RNAi target fragment for VEX2 (Tb927.11.13380, $471 \mathrm{bp}$ ) was amplified and cloned into pRPaisL45. The VEX1 (Tb927.11.16920, $574 \mathrm{bp})^{17}$ and CAF-1b (Tb927.10.7050, $458 \mathrm{bp})^{15}$ RNAi cassettes were excised prior to electroporation by digesting with $A s c$ I. For epitope-tagging at the native locus, a $710 \mathrm{bp}$ fragment of VEX2 was amplified and cloned into pNAT ${ }^{\text {TAGx45 }}$ to add an $N$-terminal $6 \times$ c-myc or GFP-tag and a fragment of $918 \mathrm{bp}$ was amplified and cloned into pNAT ${ }^{\mathrm{xTAG}} 45$ to add a C-terminal GFP tag. The vectors were linearised with XhoI and $\mathrm{HpaI}$, respectively. The VEX1 ${ }^{12 m y c 17}$ and CAF- $1 b^{12 m y c 15} C$-terminal tagging vectors were linearised with $S p h \mathrm{I}$ and NdeI, respectively. The VEX1 and CAF-1b GFP Cterminal tagging vectors were made by replacing the $12 \times \mathrm{c}$-myc tag and were also linearised with SphI and NdeI, respectively. The VEX1 C-terminal myc-tag overexpression cassette ${ }^{17}$ was excised prior to electroporation by digesting with AscI. Linearised RNAi and overexpression constructs, under the control of tetracyclineinducible promoters, were transfected into $2 \mathrm{~T} 1$ cells, which allow for targeting to a single genomic locus validated for robust inducible expression ${ }^{43}$.

ChIP-seq. ChIP and cell lysis ${ }^{46}$ was carried out with the following modifications Briefly, $2 \times 10^{8} \mathrm{~T}$. brucei bloodstream form cells expressing a $C$-terminal 12 -myc tagged endogenous copy of VEX1 were cross-linked with $1 \%$ formaldehyde for 20 min at RT. DNA was sonicated using a Bioruptor (Diagenode) with sonication beads (Diagenode, C01020031) for 5 cycles of $30 \mathrm{~s}$ on/30 s off. $C$-terminal 12-myc VEX1 was immunoprecipitated with $\alpha$-Myc antibody coupled to Dynabeads Protein $\mathrm{G}(2.8 \mu \mathrm{m})$. Antibody coupling to the Dynabeads was carried out according to the manufactures recommendations. The beads were then washed with RIPA buffer (50 mM HEPES-KOH, pKa 7.55, $500 \mathrm{mM} \mathrm{LiCl,} 1$ mM EDTA, 1.0\% NP-40, $0.7 \% \mathrm{Na}$-Deoxycholate) and eluted DNA was purified by phenol chloroform extraction and ethanol precipitation. Reads were mapped to bloodstream VSG-ESs 7 and metacyclic VSG-ESs ${ }^{47,48}$ from the Lister 427 strain. Bowtie 2-mapping ${ }^{49}$ was with the parameters --very-sensitive --no-discordant --phred33, and with a MapQ value of $>1^{18}$. Alignment files were manipulated with SAMtools ${ }^{50}$. Alignments were inspected with the Artemis genome browser ${ }^{51}$. Telomeric VSG coding sequences with a mapping quality filtering of $>1$ were aligned using deeptools computeMatrix scale-regions. The bedgraph for $\log _{2}$ fold change was generated using deepTools $2^{52}$ with $1 \mathrm{~kb}$ bins and the option smoothLength 5000, plotted in Excel and further assembled in Illustrator. The heat map was generated using deepTools. Reads were counted using BEDTools ${ }^{53}$, counting reads in non-overlapping $1 \mathrm{~kb}$ bins. Files were normalised by counting total read counts per library prior to fold change calculation for each bin. Locus maps were generated by exporting vector graphics views or regions of interest from Artemis genome browser and manipulation in Adobe illustrator. The box plot was generated in Graphpad Prism v7.0.

Affinity-enrichment of VEX1-interactors. $1 \times 10^{10}$ insect or bloodstream form $T$ brucei cells with or without a C-terminal GFP-tagged endogenous copy of VEX1 were washed three times in ice cold PBS with protease inhibitors (Roche, EDTA free) and subsequently cryomilled into a fine grindate, in a planetary ball mill (Retsch) ${ }^{20}$; protein-protein interactions are effectively preserved under these conditions. Six aliquots of $50 \mathrm{mg}$ of frozen grindate were thawed into ice cold lysis buffer (20 mM HEPES, pH 7.4, $1 \mathrm{mM} \mathrm{MgCl} 2,10 \mu \mathrm{M} \mathrm{CaCl}_{2}$, protease inhibitor cocktail) containing either $100 \mathrm{mM} \mathrm{NaCl}$ or $250 \mathrm{mM}$ citrate and either $0.1 \%$ Tween, $0.1 \%$ Brij58, $0.1 \%$ Triton X-100 or $0.1 \%$ Chaps, and pipetted to homogenise. The samples were then subjected to sonication (three pulses at $60 \% \mathrm{amp}$ ) and placed on ice. Samples were spun for $10 \mathrm{~min}$ at $20,000 \mathrm{~g}$ at $4{ }^{\circ} \mathrm{C}$ to pellet the debris. The supernatant was removed and added to a $1.5 \mathrm{ml}$ eppendorf containing dimeric $\alpha$-GFP nanobodies ${ }^{54}$ conjugated to magnetic beads (Dynabeads-Thermo Scientific) and agitated at $4{ }^{\circ} \mathrm{C}$ for $2 \mathrm{~h}$. The samples were then placed on a magnetic rack and washed three times with ice-cold lysis buffer. Samples were eluted with a non-reducing sodium dodecyl sulphate polyacrylamide gel electrophoresis (SDSPAGE) loading buffer or equivalent. The resulting proteins were fractionated by $1 D$ SDS-PAGE and visualised by silver staining.

Quantitative mass spectrometry. Affinity-enriched samples were run $2 \mathrm{~cm}$ into a NuPAGE ${ }^{\circledast}$ Novex Bis-Tris $10 \%$ gel with NuPAGE ${ }^{\circledast}$ MOPS SDS running buffer (Life Technologies) and subjected to overnight $(16 \mathrm{~h})$ trypsin digestion (Modified Sequencing Grade, Roche). Peptides were then extracted, dried in a SpeedVac (Thermo Scientific), resuspended in $50 \mu \mathrm{l} 1 \%$ formic acid, centrifuged and transferred to high-performance liquid chromatography (HPLC) vials. $5 \mu$ was typically analysed on a Q Exactive HF Hybrid Quadrupole-Orbitrap Mass Spectrometer 
(Thermo Scientific) coupled to an UltiMate 3000 RSLCnano ultra HPLC system (Thermo Scientific) and EasySpray column $(75 \mu \mathrm{m} \times 50 \mathrm{~cm}$, PepMap RSLC C18 column, $2 \mu \mathrm{m}, 100 \AA$, Thermo Scientific). The mass spectrometer was operated in data dependent mode with a single MS survey scan from 335 to $1800 \mathrm{~m} / \mathrm{z}$ followed by 20 sequential $\mathrm{m} / \mathrm{z}$ dependent MS2 scans. The 20 most intense precursor ions were sequentially fragmented by higher energy collision dissociation. The MS1 isolation window was set to $1.4 \mathrm{Da}$ and the resolution set at 60,000 . MS2 resolution was set at 15,000. The AGC targets for MS1 and MS2 were set at $3 \mathrm{e}^{6}$ ions and $2 \mathrm{e}^{5}$ ions, respectively. The normalised collision energy was set at $27 \%$. The maximum ion injection times for MS1 and MS2 were set at 50 and $19 \mathrm{~ms}$, respectively. The peptides from each fraction were separated using a mix of buffer A $(0.1 \%$ formic acid in MS grade water) and $\mathrm{B}\left(0.08 \%\right.$ formic acid in $80 \%$ MS grade $\left.\mathrm{CH}_{3} \mathrm{CN}\right)$ and eluted from the column using a flow rate of $300 \mathrm{nl} \mathrm{min}^{-1}$ and a linear gradient from 5 to $40 \%$ buffer B over $68 \mathrm{~min}$. The column temperature was set at $50^{\circ} \mathrm{C}$. RAW data files were extracted and converted to Mascot generic files (.mgf) using MSC Convert. Data were searched against the MaxQuantTbrucei database using the Mascot Search Engine (Mascot Daemon Version 2.3.2). Fold-change, relative to a sample lacking GFP-tagged VEX1, was used to derive an enrichment score. For VSG analysis, glycosylphosphatidylinositol (GPI)-specific phospholipase C (GPIPLC) cleaved soluble VSG (sVSG) was prepared and subjected to quantitative mass spectrometry analysis 17,55 ; the eluate was concentrated on an Amicon Ultra $0.5 \mathrm{ml}$ centrifugal filter (Millipore), and recovered in $100 \mu \mathrm{l}$ of water. emPAI scores are proportional to protein content in a protein mixture ${ }^{56}$.

Co-Immuniprecipitation. $4 \times 10^{8}$ bloodstream form $T$. brucei cells with or without a $C$-terminal GFP-tagged endogenous copy of VEX2 and a $C$-terminal 12-myctagged endogenous copy of CAF- $1 b$ were washed three times in ice-cold PBS with protease inhibitors and lysed into ice cold lysis buffer (RIPA, $1 \mathrm{mM}$ DTT, protease inhibitor cocktail). Pipetting to homogenise, $30 \mathrm{~s}$ of vortexing and incubation for $30 \mathrm{~min}$ at $4^{\circ} \mathrm{C}$ facilitated lysis. Samples were spun for $10 \mathrm{~min}$ at $20,000 \mathrm{~g}$ at $4^{\circ} \mathrm{C}$ to pellet debris. The supernatant was removed and added to a $1.5 \mathrm{ml}$ eppendorf containing $\alpha$-GFP antibody (Abcam) conjugated to magnetic Dynabeads and agitated at $4{ }^{\circ} \mathrm{C}$ for $2 \mathrm{~h}$. The samples were then placed on a magnetic rack and washed five times with ice-cold lysis buffer. Samples were eluted with a reducing NuPAGE LDS loading buffer. The resulting proteins were fractionated by ID SDSPAGE and analysed by protein-blotting.

Protein blotting. Protein samples were run according to standard protein separation procedures, using SDS-PAGE. However, for VEX2 detection, the use of Bis-Tris gels with a neutral $\mathrm{pH}$ environment and a bis-tris/bicine based transfer buffer (containing a reducing agent and $10 \%$ methanol) were critical for protein separation and transfer, respectively (NuPAGE, Invitrogen). Otherwise, western blotting was carried out according to standard protocols. The following primary antibodies were used: rabbit $\alpha$-VEX2 (1:1000), rabbit $\alpha$-pol-I largest subunit $^{17}(1: 500)$, rabbit $\alpha$-VSG-2 $(1: 20,000)$, rabbit $\alpha$-VSG-6 $(1: 20,000)$, mouse $\alpha$-c-myc (Millipore, clone 4A6, 1:7,000), rabbit $\alpha$-GFP (Abcam, 1:1,000) and mouse $\alpha$-EF1a (Millipore, clone CBP-KK1, 1:20,000). We used horseradish peroxidase coupled secondary antibodies ( $\alpha$-mouse and $\alpha$-rabbit, Biorad, 1:2000). Blots were developed using an enhanced chemiluminescence kit (Amersham) according to the manufacturer's instructions. Densitometry was performed using Fiji v. 2.0.0.

Microscopy. Immunofluorescence microscopy was carried out according to standard protocols, using 12 -well $5 \mathrm{~mm}$ (Thermo Scientific) or 18-well $\mu$-slides (Ibidi) for wide field, and confocal microscopy, respectively. For super-lowest speed; pinhol microscopy, the cells were attached to poly-L-lysine treated coverslips (\#1.5), stained and only then mounted onto glass slides. For colocalisation studies with pol-I we used antigen-retrieval. Prior to permeabilization, fixed cells were rehydrated in phosphate-buffered saline (PBS) for $5 \mathrm{~min}$ at RT, held at $95^{\circ} \mathrm{C}$ for 60 $\mathrm{s}$ in freshly prepared antigen-retrieval buffer $(100 \mathrm{mM}$ Tris, $5 \%$ urea, $\mathrm{pH} 9.5)$ and then washed $3 \times 5 \mathrm{~min}$ in PBS at RT. Cells were mounted in Vectashield with DAPI (wide field) or stained with $1 \mu \mathrm{g} \mathrm{ml}^{-1}$ DAPI for $10 \mathrm{~min}$ and then mounted in Vectashield without DAPI (confocal and super resolution). In T. brucei, DAPIstained nuclear and mitochondrial DNA were used as cytological markers for cellcycle stage; one nucleus and one kinetoplast $(1 \mathrm{~N}: 1 \mathrm{~K})$ indicates $\mathrm{G} 1$, one nucleus and an elongated kinetoplast $(1 \mathrm{~N}: \mathrm{eK})$ indicates $\mathrm{S}$-phase, one nucleus and two kinetoplasts $(1 \mathrm{~N}: 2 \mathrm{~K})$ indicates $\mathrm{G} 2 / \mathrm{M}$ and two nuclei and two kinetoplasts $(2 \mathrm{~N}: 2 \mathrm{~K})$ indicates post-mitosis ${ }^{57,58}$. Primary antisera were rat $\alpha$-VSG-2 $(1: 10,000)$, rabbit $\alpha$ VSG-6 $(1: 10,000)$, rabbit $\alpha$-VSG-13 ${ }^{59}(1: 5000)$, mouse $\alpha$-VSG- $3^{60}$ coupled with Alexa 488 (1:500), mouse $\alpha$-EP procyclin (Cedarlene, 1:1,000), rabbit $\alpha$-GFP (Invitrogen, 1:250; Abcam, 1:500), mouse $a$-myc (Source Bioscience, clone 9E10, 1:200 or New England Biolabs, clone 9B11, 1:2,000), rabbit $\alpha$-pol-I largest subunit ${ }^{17}$ (1:100), rabbit $\alpha$-NOG1 ${ }^{61}$ (1:500) or rabbit $\alpha$-histone H3 (Abcam, 1:1000). The secondary antibodies were Alexa Fluor conjugated goat antibodies (Thermo Scientific): $\alpha$-mouse, $\alpha$-rat and $\alpha$-rabbit, AlexaFluor 488 or Alexa Fluor 568 (1:1000 or $1: 2000$, for confocal or wide field field microscopy, respectively) or a-rat Alexa 647 (1:1000). For the colocalisation studies in Fig. 8a, b and the quantification in Fig. $5 c$, cells were analysed by confocal microscopy, using a Leica TCS SP8 confocal laser scanning microscope and the Leica Application Suite X (LASX) software
(Leica, Germany). For the remaining quantifications, cells were analysed by wide field microscopy, using a Zeiss Axiovert $200 \mathrm{M}$ microscope with an AxioCam MRm camera and the ZEN Pro software (Carl Zeiss, Germany). The images were acquired as $z$-stacks $(0.1-0.2 \mu \mathrm{m})$ and in the case of the wife field microscopy, further deconvolved using the fast iterative algorithm in Zen Pro. For all quantifications, images were acquired with the same settings and equally processed. Corrected total nuclear fluorescence $=$ integrated density $-($ area of selected nucleus $\times$ mean fluorescence of background readings). Representative images were further acquired using a Leica TCS SP8 confocal laser scanning microscope in Hyvolution Mode and the Leica Application Suite X (LASX) software (Leica, Germany). The Hyvolution mode allows super-resolution level images, with settings: highest resolution/lowest speed; pinhole 0.5-Images with DNA in grey (or cyan); Figs. 1d-f, 2a, b, 3c (right hand side) 4f, 5c (right hand side), 6c, 7b, 8a, b (right hand side), 9a, b and Supplementary Fig. 3c, d. All the confocal images (with or without Hyvolution mode) correspond to maximal 3D projections by brightest intensity stacks of approximately 30 slices of $0.1 \mu \mathrm{m}$. All the images were processed, scored and the signal quantified using Fiji v. 2.0.0 $0^{62}$. Actinomycin D was applied at $10 \mu \mathrm{g} \mathrm{ml}^{-1}$ for $30 \mathrm{~min}$ and pol-I inhibitor (BMH-21) at $1 \mu \mathrm{M}$ for $30 \mathrm{~min}$, both at $37^{\circ} \mathrm{C}$.

Flow cytometry. Flow cytometry was carried out according to standard procedures. The primary antibodies were as follows: rat $\alpha$-VSG-2 $(1: 10,000)$ and rabbit $\alpha$-VSG-6 $(1: 10,000)$. Secondary antibodies were goat $\alpha$-rat Alexa Fluor 647 and goat $a$-rabbit Alexa Fluor 488 (both 1:2000). DNA was stained with propidium iodide at $10 \mu \mathrm{g} \mathrm{ml}^{-1}$. Samples were analysed on a BD LSRFortessa (BD Biosciences) and data were visualised using FlowJo software. More than 40,000 events were analysed to determine the percentage of cells in each quadrant.

Transcriptome analysis. RNA-seq analysis was performed using $2 \mathrm{~T} 1$ cells and uninduced or induced clones of VEX2 (24 and $48 \mathrm{~h})$, VEX1/VEX2 (24 and $48 \mathrm{~h}$ ) or CAF-1b (24 h) RNAi. Briefly, polyadenylated transcripts were enriched using poly$\mathrm{dT}$ beads and reverse-transcribed before sequencing on a HiSeq platform (Illumina). Reads were mapped to a hybrid assembly consisting of the T. brucei 927 reference genome plus the bloodstream VSG-ESs ${ }^{7}$ and metacyclic VSG-ESs ${ }^{47,48}$ from the Lister 427 strain. Bowtie 2-mapping ${ }^{49}$ was with the parameters --verysensitive --no-discordant --phred33. Alignment files were manipulated with SAMtools ${ }^{50}$. Per-gene read counts were derived using the Artemis genome browser ${ }^{6}, \mathrm{MapQ}>1^{18}$. Read counts were normalised using edge $\mathrm{R}^{64}$ and differential expression was determined with classic edgeR. RPKM values were derived from normalised read counts in edgeR. Base pair resolution plots were generated using an in-house script.

Statistical analysis. All statistical analyses were performed using GraphPad Prism Software (version 7.0), except the transcriptomic analysis (described above). Detailed information regarding replicates, statistical tests and outputs can be found in Supplementary Data 1 , sheet 6.

Resources and reagents. Details of resources and reagents can be found in Supplementary Data 1, sheet 7 . All unique materials are available on request.

Reporting summary. Further information on research design is available in the Nature Research Reporting Summary linked to this article.

\section{Data availability}

RNA-seq and ChIP-seq data have been deposited in the European Nucleotide Archive www.ebi.ac.uk/ena (Accession nos. PRJEB21615 and PRJEB25352, respectively). Proteomics data have been deposited in the PRoteomics IDEntifications (PRIDE) database www.ebi.ac.uk/pride, ProteomeXchange accession no. PXD013304. The source data underlying Figs. 1d, 2a, b, 3a-e, 4f, 5a-e, 6a-c, 7a, 8a, b, 9a-c, 10a, b, Supplementary Figs. 3a-f, 4c, 6a-c, $8 \mathrm{a}, \mathrm{b}, \mathrm{d}$ are provided as a Source Data file.

Received: 23 October 2018 Accepted: 4 June 2019

Published online: 09 July 2019

\section{References}

1. Guizetti, J. \& Scherf, A. Silence, activate, poise and switch! Mechanisms of antigenic variation in Plasmodium falciparum. Cell. Microbiol. 15, 718-726 (2013).

2. Gargantini, P. R., Serradell Mdel, C., Rios, D. N., Tenaglia, A. H. \& Lujan, H. D. Antigenic variation in the intestinal parasite Giardia lamblia. Curr. Opin. Microbiol. 32, 52-58 (2016).

3. Horn, D. Antigenic variation in African trypanosomes. Mol. Biochem Parasitol. 195, 123-129 (2014). 
4. Monahan, K. \& Lomvardas, S. Monoallelic expression of olfactory receptors. Annu Rev. Cell Dev. Biol. 31, 721-740 (2015).

5. Vettermann, C. \& Schlissel, M. S. Allelic exclusion of immunoglobulin genes: models and mechanisms. Immunol. Rev. 237, 22-42 (2010).

6. Kassem, A., Pays, E. \& Vanhamme, L. Transcription is initiated on silent variant surface glycoprotein expression sites despite monoallelic expression in Trypanosoma brucei. Proc. Natl Acad. Sci. USA 111, 8943-8948 (2014).

7. Hertz-Fowler, C. et al. Telomeric expression sites are highly conserved in Trypanosoma brucei. PLoS ONE 3, e3527 (2008).

8. Chaves, I. et al. Subnuclear localization of the active variant surface glycoprotein gene expression site in Trypanosoma brucei. Proc. Natl Acad. Sci. USA 95, 12328-12333 (1998)

9. Navarro, M. \& Gull, K. A pol I transcriptional body associated with VSG mono-allelic expression in Trypanosoma brucei. Nature 414, 759-763 (2001).

10. Figueiredo, L. M. \& Cross, G. A. Nucleosomes are depleted at the VSG expression site transcribed by RNA polymerase I in African trypanosomes. Eukaryot. Cell 9, 148-154 (2010).

11. Stanne, T. M. \& Rudenko, G. Active VSG expression sites in Trypanosoma brucei are depleted of nucleosomes. Eukaryot. Cell 9, 136-147 (2010).

12. Aresta-Branco, F., Pimenta, S. \& Figueiredo, L. M. A transcriptionindependent epigenetic mechanism is associated with antigenic switching in Trypanosoma brucei. Nucleic Acids Res. 44, 3131-3146 (2015).

13. Narayanan, M. S. \& Rudenko, G. TDP1 is an HMG chromatin protein facilitating RNA polymerase I transcription in African trypanosomes. Nucleic Acids Res. 41, 2981-2992 (2013)

14. Lopez-Farfan, D., Bart, J. M., Rojas-Barros, D. I. \& Navarro, M. SUMOylation by the E3 ligase TbSIZ1/PIAS1 positively regulates VSG expression in Trypanosoma brucei. PLoS Pathog. 10, e1004545 (2014).

15. Alsford, S. \& Horn, D. Cell-cycle-regulated control of VSG expression site silencing by histones and histone chaperones ASF1A and CAF-1b in Trypanosoma brucei. Nucleic Acids Res. 40, 10150-10160 (2012).

16. Muller, L. S. M. et al. Genome organization and DNA accessibility control antigenic variation in trypanosomes. Nature 563, 121-125 (2018).

17. Glover, L., Hutchinson, S., Alsford, S. \& Horn, D. VEX1 controls the allelic exclusion required for antigenic variation in trypanosomes. Proc. Natl Acad. Sci. USA 113, 7225-7230 (2016).

18. Hutchinson, S., Glover, L. \& Horn, D. High-resolution analysis of multi-copy variant surface glycoprotein gene expression sites in African trypanosomes. BMC Genom. 17, 806 (2016)

19. Callejas, S., Leech, V., Reitter, C. \& Melville, S. Hemizygous subtelomeres of an African trypanosome chromosome may account for over $75 \%$ of chromosome length. Genome Res. 16, 1109-1118 (2006).

20. Obado, S. O., Field, M. C., Chait, B. T. \& Rout, M. P. High-efficiency isolation of nuclear envelope protein complexes from trypanosomes. Methods Mol. Biol. 1411, 67-80 (2016).

21. He, F. \& Jacobson, A. Nonsense-mediated mRNA decay: degradation of defective transcripts is only part of the story. Annu. Rev. Genet. 49, 339-366 (2015).

22. Delhi, P., Queiroz, R., Inchaustegui, D., Carrington, M. \& Clayton, C. Is there a classical nonsense-mediated decay pathway in trypanosomes? PLoS ONE 6, e25112 (2011)

23. Lykke-Andersen, S. \& Jensen, T. H. Nonsense-mediated mRNA decay: an intricate machinery that shapes transcriptomes. Nat. Rev. Mol. Cell Biol. 16, 665-677 (2015).

24. Hammond, C. M., Stromme, C. B., Huang, H., Patel, D. J. \& Groth, A. Histone chaperone networks shaping chromatin function. Nat. Rev. Mol. Cell Biol. 18, 141-158 (2017).

25. Kerry, L. E. et al. Selective inhibition of RNA polymerase I transcription as a potential approach to treat African trypanosomiasis. PLoS Negl. Trop. Dis. 11, e0005432 (2017).

26. Alsford, S. et al. High-throughput phenotyping using parallel sequencing of RNA interference targets in the African trypanosome. Genome Res. 21, 915-924 (2011).

27. Aitcheson, N. et al. VSG switching in Trypanosoma brucei: antigenic variation analysed using RNAi in the absence of immune selection. Mol. Microbiol 57, 1608-1622 (2005).

28. Devlin, R. et al. Mapping replication dynamics in Trypanosoma brucei reveals a link with telomere transcription and antigenic variation. Elife 5, e12765 (2016)

29. Mendell, J. T., ap Rhys, C. M. \& Dietz, H. C.Separable roles for rent1/hUpf1 in altered splicing and decay of nonsense transcripts. Science 298, 419-422 (2002).

30. Azzalin, C. M., Reichenbach, P., Khoriauli, L., Giulotto, E. \& Lingner, J. Telomeric repeat containing RNA and RNA surveillance factors at mammalian chromosome ends. Science 318, 798-801 (2007).

31. Ciaudo, C. et al. Nuclear mRNA degradation pathway(s) are implicated in Xist regulation and X chromosome inactivation. PLoS Genet 2, e94 (2006).
32. Hnisz, D., Shrinivas, K., Young, R. A., Chakraborty, A. K. \& Sharp, P. A. A phase separation model for transcriptional control. Cell 169, 13-23 (2017).

33. Nunes, V. S. \& Moretti, N. S. Nuclear subcompartments: an overview. Cell Biol. Int. 41, 2-7 (2017).

34. Ramachandran, S. \& Henikoff, S. Transcriptional regulators compete with nucleosomes post-replication. Cell 165, 580-592 (2016).

35. Radman-Livaja, M. et al. Patterns and mechanisms of ancestral histone protein inheritance in budding yeast. PLoS Biol. 9, e1001075 (2011).

36. Francis, N. J., Follmer, N. E., Simon, M. D., Aghia, G. \& Butler, J. D. Polycomb proteins remain bound to chromatin and DNA during DNA replication in vitro. Cell 137, 110-122 (2009).

37. Kadauke, S. et al. Tissue-specific mitotic bookmarking by hematopoietic transcription factor GATA1. Cell 150, 725-737 (2012).

38. Cheloufi, S. et al. The histone chaperone CAF-1 safeguards somatic cell identity. Nature 528, 218-224 (2015).

39. Loyola, A. et al. The HP1a-CAF1-SetDB1-containing complex provides $\mathrm{H} 3 \mathrm{~K} 9$ mel for Suv39-mediated K9me3 in pericentric heterochromatin. EMBO Rep. 10, 769-775 (2009).

40. Lee, S. B., Ou, D. S., Lee, C. F. \& Juan, L. J. Gene-specific transcriptional activation mediated by the p150 subunit of the chromatin assembly factor $1 . \mathrm{J}$. Biol. Chem. 284, 14040-14049 (2009).

41. Sim, C. K., Perry, S., Tharadra, S. K., Lipsick, J. S. \& Ray, A. Epigenetic regulation of olfactory receptor gene expression by the Myb-MuvB/dREAM complex. Genes Dev. 26, 2483-2498 (2012).

42. Landeira, D., Bart, J. M., Van Tyne, D. \& Navarro, M. Cohesin regulates VSG monoallelic expression in trypanosomes. J. Cell Biol. 186, 243-254 (2009).

43. Alsford, S., Kawahara, T., Glover, L. \& Horn, D. Tagging a T. brucei RRNA locus improves stable transfection efficiency and circumvents inducible expression position effects. Mol. Biochem. Parasitol. 144, 142-148 (2005).

44. Glover, L. et al. Genome-scale RNAi screens for high-throughput phenotyping in bloodstream-form African trypanosomes. Nat. Protoc. 10, 106-133 (2015).

45. Alsford, S. \& Horn, D. Single-locus targeting constructs for reliable regulated RNAi and transgene expression in Trypanosoma brucei. Mol. Biochem. Parasitol. 161, 76-79 (2008).

46. Siegel, T. N. et al. Four histone variants mark the boundaries of polycistronic transcription units in Trypanosoma brucei. Genes Dev. 23, 1063-1076 (2009).

47. Cross, G. A., Kim, H. S. \& Wickstead, B. Capturing the variant surface glycoprotein repertoire (the VSGnome) of Trypanosoma brucei Lister 427. Mol. Biochem. Parasitol. 195, 59-73 (2014).

48. Kolev, N. G., Ramey-Butler, K., Cross, G. A., Ullu, E. \& Tschudi, C. Developmental progression to infectivity in Trypanosoma brucei triggered by an RNA-binding protein. Science 338, 1352-1353 (2012).

49. Langmead, B. \& Salzberg, S. L. Fast gapped-read alignment with Bowtie 2. Nat. Methods 9, 357-359 (2012).

50. Li, $\mathrm{H}$. et al. The sequence alignment/Map format and SAMtools. Bioinformatics 25, 2078-2079 (2009).

51. Rutherford, K. et al. Artemis: sequence visualization and annotation. Bioinformatics 16, 944-945 (2000).

52. Ramirez, F. et al. deepTools2: a next generation web server for deepsequencing data analysis. Nucleic Acids Res. 44, W160-W165 (2016).

53. Quinlan, A. R. \& Hall, I. M. BEDTools: a flexible suite of utilities for comparing genomic features. Bioinformatics 26, 841-842 (2010).

54. Fridy, P. C. et al. A robust pipeline for rapid production of versatile nanobody repertoires. Nat. Methods 11, 1253-1260 (2014).

55. Manthri, S., Guther, M. L., Izquierdo, L., Acosta-Serrano, A. \& Ferguson, M. A. Deletion of the TbALG3 gene demonstrates site-specific $\mathrm{N}$-glycosylation and $\mathrm{N}$ glycan processing in Trypanosoma brucei. Glycobiology 18, 367-383 (2008)

56. Ishihama, Y. et al. Exponentially modified protein abundance index (emPAI) for estimation of absolute protein amount in proteomics by the number of sequenced peptides per protein. Mol. Cell Proteom. 4, 1265-1272 (2005).

57. Siegel, T. N., Hekstra, D. R. \& Cross, G. A. Analysis of the Trypanosoma brucei cell cycle by quantitative DAPI imaging. Mol. Biochem. Parasitol. 160, 171-174 (2008).

58. Woodward, R. \& Gull, K. Timing of nuclear and kinetoplast DNA replication and early morphological events in the cell cycle of Trypanosoma brucei. J. Cell Sci. 95, 49-57 (1990)

59. Figueiredo, L. M., Janzen, C. J. \& Cross, G. A. A histone methyltransferase modulates antigenic variation in African trypanosomes. PLoS Biol. 6, e161 (2008).

60. Pinger, J., Chowdhury, S. \& Papavasiliou, F. N. Variant surface glycoprotein density defines an immune evasion threshold for African trypanosomes undergoing antigenic variation. Nat. Commun. 8, 828 (2017).

61. Park, J. H., Jensen, B. C., Kifer, C. T. \& Parsons, M. A novel nucleolar G-protein conserved in eukaryotes. J. Cell Sci. 114, 173-185 (2001).

62. Schindelin, J. et al. Fiji: an open-source platform for biological-image analysis Nat. Methods 9, 676-682 (2012). 
63. Carver, T., Harris, S. R., Berriman, M., Parkhill, J. \& McQuillan, J. A. Artemis: an integrated platform for visualization and analysis of high-throughput sequence-based experimental data. Bioinformatics 28, 464-469 (2012).

64. Robinson, M. D., McCarthy, D. J. \& Smyth, G. K. edgeR: a Bioconductor package for differential expression analysis of digital gene expression data. Bioinformatics 26, 139-140 (2010).

\section{Acknowledgements}

We thank T. Owen-Hughes, N. Wiechens and V. Singh for assistance with ChIP-seq library preparation; A. Cassidy for Illumina sequencing; R. Clark of the Flow Cytometry and Cell Sorting Facility at the University of Dundee for cell sorting; D. Lamont, K. Beattie and S. Kosto of the FingerPrints Proteomics Facility at the University of Dundee for assistance with quantitative proteomics; the Dundee Imaging Facility; J. Rouse for access to the Leica Confocal SP8 Hyvolution microscope; S. Alsford (London School of Hygiene \& Tropical Medicine) for the CAF-1 RNAi and CAF-1 tagging constructs; Marilyn Parsons (University of Washington) for the $\alpha$-NOG1 antisera and C. Marques for discussions. The work was funded by Wellcome Trust Investigator Awards to D.H. [100320/Z/12/Z] and M.C.F. [204697/Z/16/Z] with additional support from a Wellcome Trust Centre Award [203134/Z/16/Z]. The University of Dundee Flow Cytometry and Imaging Facilities are supported by a Wellcome Trust award [097418/Z/11/Z], and the MRC Next Generation Optical Microscopy award [MR/K015869/1], respectively; while both the Proteomics and Imaging Facilities are supported by a Wellcome Trust Technology Platform award [097945/B/11/Z]

\section{Author contributions}

Conceptualisation: J.F., L.G., M.C.F. and D.H. Data curation: J.F. and S.H. Investigation: J.F., L.G. and C.B. Supervision: D.H. Original draft: J.F. and D.H. Review and editing: J.F., L.G., S.H., M.C.F. and D.H.

\section{Additional information}

Supplementary Information accompanies this paper at https://doi.org/10.1038/s41467019-10823-8.

Competing interests: The authors declare no competing interests.

Reprints and permission information is available online at http://npg.nature.com/ reprintsandpermissions/

Peer review information: Nature Communications thanks Luisa Figueiredo, Sanjeeva Srivastava and the other, anonymous, reviewer(s) for their contribution to the peer review of this work. Peer reviewer reports are available.

Publisher's note: Springer Nature remains neutral with regard to jurisdictional claims in published maps and institutional affiliations.

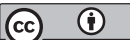

Open Access This article is licensed under a Creative Commons Attribution 4.0 International License, which permits use, sharing, adaptation, distribution and reproduction in any medium or format, as long as you give appropriate credit to the original author(s) and the source, provide a link to the Creative Commons license, and indicate if changes were made. The images or other third party material in this article are included in the article's Creative Commons license, unless indicated otherwise in a credit line to the material. If material is not included in the article's Creative Commons license and your intended use is not permitted by statutory regulation or exceeds the permitted use, you will need to obtain permission directly from the copyright holder. To view a copy of this license, visit http://creativecommons.org/ licenses/by/4.0/.

(C) The Author(s) 2019 